UFIFT-HEP-97-29; UMD-PP-98-54

hep-ph/9712516, December 1997

\title{
A Family-Universal Anomalous $U(1)$ in String Models as the Origin of Supersymmetry Breaking and Squark Degeneracy
}

\author{
Alon E. Faraggi ${ }^{1}$ and Jogesh C. Pati ${ }^{2}$ \\ ${ }^{1}$ Department of Physics, University of Florida, Gainesville, FL 32611 USA \\ 2 Department of Physics, University of Maryland, College park, MD 20742
}

\begin{abstract}
Recently a promising mechanism for supersymmetry breaking that utilizes both an anomalous $U(1)$ gauge symmetry and an effective mass term $m \sim 1 \mathrm{TeV}$ of certain relevant fields has been proposed. In this paper we examine whether such a mechanism can emerge in superstring derived free fermionic models. We observe that certain three generation string solutions, though not all, lead to an anomalous $U(1)$ which couples universally to all three families. The advantages of this three-family universality of $U(1)_{A}$, compared to the twofamily case, proposed in earlier works, in yielding squark degeneracy, while avoiding radiative breaking of color and charge, are noted. The root cause of the flavor universality of $U(1)_{A}$ is the cyclic permutation symmetry that characterizes the $Z_{2} \times Z_{2}$ orbifold compactification with standard embedding, realized in the free fermionic models by the NAHE set. It is shown that nonrenormalizable terms which contain hidden-sector condensates, generate the required suppression of the relevant mass term $m$, compared to the Planck scale. While the $D$-term of the family universal $U(1)_{A}$ leads to squark degeneracy, those of the family dependent $U(1)$ 's, remarkably enough, are found to vanish for the solutions considered, owing to minimization of the potential.
\end{abstract}




\section{Introduction}

Understanding the origin of (i) supersymmetry breaking and simultaneously (ii) of the extreme degeneracy in the masses of the squarks in at least the first two families, as inferred from the miniscule strengths of the $K^{0}-\bar{K}^{0}$ transition, is still among the important unsettled issues in particle physics. Equally important is understanding the large hierarchy between the Planck scale and the SUSY breaking mass splitting $\delta m_{s}$, reflected by the ratio $\delta m_{s} / M_{\text {Planck }} \sim 10^{-15}$.

Several mechanism have been proposed to implement SUSY-breaking. These include the ideas of: (i) gaugino condensation in the hidden sector [1]; (ii) dilaton dominated SUSY breaking (DDSB) [2], (iii) gauge mediated SUSY breaking (GMSB) [3]; whose intrinsic origin is delegated to an unknown mechanism involving an effective singlet field, which couples to a set of messenger particles; and (iv) SUSY- breaking, induced through joint effects of an anomalous $U(1)$ gauge interaction and effective mass-terms of certain relevant fields, which carry the anomalous $U(1)$ charge $4,5,6$. The mass terms in case (iv) represent the scale of SUSY-breaking mass splitting $\delta m_{s}$, and thus, on phenomenological grounds, they must be of order $1 \mathrm{TeV}$.

Among these, the mechanism of DDSB automatically yields squark degeneracy at the tree level. It however has the problem of possible color and charge breaking (see e.g. the last paper in ref. [2]). The GMSB yields squark degeneracy, provided that the superpotential Yukawa interactions of the messenger fields with the Standard Model fields are suppressed. The existence of such messenger fields in superstring derived models was proposed [8]. That of the anomalous $U(1)$ can yield the desired degeneracy provided that it couples universally to at least the first two families, which is assumed in refs. [5, 7]. Short of deriving any of these from an underlying theory, such as superstring theory, however, the scale of SUSY-breaking mass splittings $\delta m_{s}$ as well as the choice of fields and of their quantum numbers are rather arbitrary. They must therefore be put in by hand.

It is thus of great interest to examine whether any of these mechanisms could in fact emerge from within a superstring theory. Now, phenomenologically viable, 
solutions of string theory invariably do indeed contain an anomalous $U(1)$ as a generic feature (see e.g. refs. [9, 10, 11, 12, 13, 14, 15], as examples of models based on the free fermionic construction [16]. We wish therefore to explore in this note the viability of supersymmetry breaking through an anomalous $U(1)$ in the context of such string derived solutions. In particular, we examine whether they can yield either a two or a three-family universal anomalous $U(1)$ that would lead to squark mass degeneracy (following SUSY-breaking) on the one hand, and yet would not conflict with the observed hierarchy in the masses of the fermions on the other hand; and whether these solutions can also yield non-vanishing but strongly suppressed mass terms $m<<M_{\text {Planck }}$ of certain relevant fields, which are essential to trigger SUSYbreaking. The smallness of $m$ compared to the Planck mass would then account for the large hierarchy between $\delta m_{s}$ and $M_{\text {Planck }}$.

Given that string theory yields a vast set of solutions at the tree level and that no guiding principle is available to choose between them, it is, of course, still premature to take too seriously any specific solution. Yet certain generic features of a class of solutions, related especially to their symmetry properties, may well survive in the final picture. With this in mind and for concreteness, we examine the issues noted above within a specific class of string-derived solutions, which are obtained in the free fermionic formulation [16], and yield non-GUT standard model-like gauge symmetries with three generations [13, 14]. Later, we will comment on the issue of flavor universality of the anomalous $U(1)$ in some other solutions such as those of refs. [9] and [11]. A priori motivations for considering the class of solutions obtained in refs [13, 14] are that (a) they seem capable of generating qualitatively the right-texture for fermion masses and mixings; (b) they provide a natural doublet-triplet splitting mechanism because of their non-GUT character and (c) they also possess extra gauge symmetries, beyond SUSY GUTs, which, together with the allowed pattern of VEVs, safeguard proton stability from all potential dangers [17, 18], including those which may arise from higher dimensional operators and from the exchange of color triplets in the heavy tower of string states. These extra symmetries also turn out to be helpful in suppressing $\nu_{L}-\tilde{H}$ mixing operator [19]. Last but not the least, having 
their origin in a string theory, they of course satisfy gauge coupling unification in spite of their non-GUT character [20]. The obvious question is whether this class of string solutions also permits supersymmetry breaking at the electroweak scale through an anomalous $U(1)$, while preserving family-universality in squark masses.

In section 2, we observe that the desired family universality of the anomalous $U(1)$ is by no means a general property of string solutions, but it holds in the class of solutions obtained in ref. [13] and [14. We point out the root cause why it holds for this class. In section 3 and 4, we discuss supersymmetry breaking and generation of relevant mass-terms for these solutions. We show in section 4 that there exist solutions in this class which yield operators of dimension $n \geq 4$, that induce highly suppressed relevant mass terms, $\sim(1 / 2-50) \mathrm{TeV}$. These mass terms, together with the anomalous $U(1)$, induce SUSY breaking. Thus these solutions have the potential for explaining (a) supersymmetry breaking; (b) gauge hierarchy and (c) squark degeneracy.

An issue of special concern is that string solutions invariably possess a host of other $U(1)$ s, which are family dependent, and contributions from their $D$-terms, if non-vanishing, could potentially spoil squark degeneracy. We show in section 3 that there exist solutions for which, the contributions from the undesirable $D$-terms, remarkably enough, vanish owing to a minimization of the potential. In short, the class of string solutions considered here, though by no means unique, possesses three non-trivial and highly desirable features: (i) a family universal anomalous $U(1)$, (ii) suitably suppressed mass terms of relevant fields which trigger SUSY breaking, and (iii) vanishing of family dependent $D$-term contributions. In section 5 , we mention certain features of phenomenological interest. In particular string solutions of ref. [13] or [14] lead to approximate squark degeneracy for all three families. This is in contrast to the case of ref. [5] and [7], where the degeneracy holds (because of the choice of the anomalous charge) only for the first two families. Advantages of three compared to two family squark-degeneracy in avoiding radiative color and electric charge breaking - is noted. We also point out that the solution of ref. [14] leads to intra-family sfermion degeneracy (i.e. $m_{\tilde{q}_{L}}=m_{\tilde{u}_{R}}=m_{\tilde{d}_{L}}=m_{\tilde{L}}$, etc.), whereas 
that of ref. [13] leads to considerable splitting between the members of a family. In the concluding section, we make some general remarks about the prospect of supersymmetry-breaking through $U(1)_{A}$, and the possibility of other contributions to supersymmetry breaking.

\section{A family universal anomalous $U(1)$ in a class of string so- lutions}

We begin by recalling certain salient features of the solutions based on the free fermionic formulation [16]. They are defined by a set of boundary condition basis vectors and the associated one-loop GSO projection coefficients. The massless states are obtained by applying the generalized GSO projections. Each massless state defines a vertex operator and the cubic and higher order terms in the superpotential are obtained by calculating the correlators between the vertex operators [21, 22].

The specific class of solutions [13, 14] which we examine here are generated by a set of eight boundary condition basis vectors. The first five of these, denoted by $\left\{\mathbf{1}, S, b_{1}, b_{2}, b_{3}\right\}$, constitute the so-called NAHE set [9, 23]. They are common to a large class of viable string solutions, including those of refs. [9] [11], and [12]. The properties of the NAHE set are crucial to understanding how the required flavor universality of the anomalous $U(1)$ may arise in certain free fermionic solutions. We therefore refer the reader to refs. [9, 23] for definition of these basis vectors and their detailed properties. Here we note only certain salient features. The vectors $\{\mathbf{1}, S\}$ give rise to a solution with $N=4$ space-time supersymmetry and $S O(44)$ gauge symmetry. The vectors $b_{1}, b_{2}$ and $b_{3}$ break $N=4$ to $N=1$ and $S O(44)$ to $S O(10) \times E_{8} \times S O(6)^{3}$, where $S O(10)$ is identified with the GUT symmetry containing the Standard Model. Each of the vectors $b_{1}, b_{2}$ and $b_{3}$ produces 16 multiplets, each of which is a 16 of $S O(10)$; thus there are altogether 48 generations. The sixteen generations produced by each $b_{j}$ are charged with respect to only one of the $S O(6)_{j}$ - symmetries, which is why the $S O(6)_{j}$ - symmetries provide the origin of flavor symmetries. Note that at this stage, there is a complete permutation symmetry 
between the sectors $b_{1}, b_{2}$ and $b_{3}$, which is reflected in the full set of gauge interactions as well as in the superpotential. It is this permutation symmetry which leads to family universality of the anomalous $U(1)$ in some models.

It is important to note that the NAHE set corresponds to a $Z_{2} \times Z_{2}$ orbifold compactification. This seemingly apparent observation has far reaching phenomenological implications. The focus in this paper is on SUSY breaking and squark degeneracy. The correspondence of the NAHE set with $Z_{2} \times Z_{2}$ orbifold compactification is best illustrated by adding to the NAHE set the boundary condition basis vector $\mathbf{X}$ with periodic boundary conditions for the world-sheet fermions $\left\{\bar{\psi}^{1, \cdots, 5}, \bar{\eta}^{1,2,3}\right\}$, and antiperiodic boundary conditions for all others. With a suitable choice of the generalized GSO projection coefficients the $S O(10)$ gauge group is enhanced to $E_{6}$. The $S O(6)^{3}$ symmetries are broken to $S O(4)^{3} \times U(1)^{3}$. One combination of the $U(1)$ symmetries is embedded in $E_{6}$. The gauge group in this case would be $E_{6} \times E_{8} \times S O(4)^{3} \times U(1)^{2}$. This extended NAHE set then corresponds to a $Z_{2} \times Z_{2}$ orbifold with the standard embedding of the gauge connection [24]. The three sectors generated by $b_{1}, b_{2}$ and $b_{3}$ are the three twisted sectors of the orbifold models. The cyclic permutation symmetry associated with the NAHE set is thus simply the symmetry between the three twisted sectors of the $Z_{2} \times Z_{2}$ orbifold, with standard embedding. The permutation symmetry also applies to the spectrum that arises from the untwisted sector, including the moduli. The phenomenological motivation for this symmetry will become apparent in the context of supersymmetry breaking and squark degeneracy. Whether this symmetry is unique to the $Z_{2} \times Z_{2}$ orbifold compactification is an open question.

The next stage in the construction of viable solutions is the introduction of additional boundary condition basis vectors, which reduce the number of chiral generations from forty-eight to three, barring possible vector-like multiplets. These also break $S O(10)$ to one of its subgroups - e.g. $S U(5) \times U(1)$ [9, $S O(6) \times S O(4)$ [11 or $S U(3) \times S U(2) \times U(1)^{2}$ [10, 13, 14]. The hidden $E_{8}$ symmetry is typically also broken to one of its subgroups, and the horizontal $S O(6)^{3}$ symmetry breaks typically to Abelian factors of $U(1)^{n}$, where $n(\geq 3)$ varies between the solutions. As we discuss 
below, the permutation symmetry of the full set of gauge interactions with respect to the three chiral families is retained for solutions of the type presented in refs [13] and [14], in spite of the introduction of three additional boundary condition basis vectors (beyond the NAHE set). But this is not the case for the solutions of refs. 99 and [11]. The reason for this difference is that in the case of ref. [13] and [14] the three chiral families have their origin entirely in the sectors $b_{1}, b_{2}$ and $b_{3}$ respectively, and there are no additional vector-like families. By contrast, for the cases of refs. [9] and [11], owing to the nature of the additional boundary condition basis vectors, there are vector-like multiplets in addition to the three chiral families, and the latter do not all arise from the sectors $b_{1}, b_{2}$ and $b_{3}$ respectively.

In summary, the NAHE set naturally gives rise to the permutation symmetry of the three families, or rather three groups of families, both in the gauge as well as in the superpotential sector. This symmetry need not, however, be retained in general in the presence of additional boundary condition basis vectors, beyond the NAHE set. It is intriguing that the stated symmetry is fully retained in the gauge sector (though it is partially lost in the superpotential, see below) for solutions of the type presented in [13 and 14]. As we will show, this permutation symmetry in the gauge sector guarantees family-universality of the anomalous $U(1)$.

As concrete examples, we consider the solutions of both ref. [13] and ref. [14], which we will refer to as solutions I and II respectively. They are very similar for most purposes, yet they possess certain crucial differences. The gauge symmetry in both cases, arising from the NS sector, after application of all the GSO projections, has the following form, at the string scale:

$$
\mathcal{G}=\left[S U(3)_{C} \times S U(2)_{L} \times U(1)_{B-L} \times U(1)_{T_{3_{R}}}\right] \times\left[G_{M}=\prod_{i=1}^{6} U(1)_{i}\right] \times G_{H}
$$

Here, $U(1)_{i}$ denote six horizontal flavor symmetries, which descend from $S O(6)^{3}$, and act non-trivially on the three chiral families, Higgs multiplets as well as Hidden matter states. In both cases, $G_{H}=S U(5)_{H} \times S U(3)_{H} \times U(1)_{H}^{2}$, is the gauge symmetry of the hidden sector. In the model of Ref. [14] additional space-time vector bosons arise from the sector $\mathbf{1}+\alpha+2 \gamma$ and enhance the $S U(3)_{C}$ gauge group to $S U(4)_{C}$ 25 
(which we use in tables 4,5 and 6). This enhancement, however, does not affect our discussion here.

The massless spectrum of solution I (ref. [13]), together with the quantum numbers of the respective states, is exhibited in tables 1,2 and 3. The spectrum includes the three generations that arise from the sectors $b_{1}, b_{2}$ and $b_{3}$, the Higgs like multiplets $h_{1,2,3}, h_{45}$ and their conjugates, the color triplets $\left(D_{45}, \bar{D}_{45}\right)$, the $S O(10)$ singlets $\Phi_{1,2,3}^{ \pm}, \Phi_{45}, \Phi_{12}, \Phi_{13}, \Phi_{23}$ and their conjugates, and the hidden sector multiplets $\left(V_{i}, \bar{V}_{i}, T_{i} \bar{T}_{i}\right)_{i=1,2,3}$. We see that each generation that arises from the sector $b_{j}$, is charged with respect to two of the $U(1)$ 's - i.e. $U(1)_{R_{j}}$ and $U(1)_{R_{j+3}}$. For each right-moving gauged $U(1)$ symmetry, there is a corresponding left-moving global $U(1)$ symmetry, denoted by $U(1)_{L_{j}}$ and $U(1)_{L_{j+3}}$, and the states from each sector $b_{j}$ are charged with respect to two of these global symmetries.

The spectrum for solution II (Ref. [14]) is very similar. The main difference is that the six $S O(10)$-singlets $\Phi_{1,2,3}^{ \pm}$are replaced by only two fields $\Phi_{1,2}$, while $h_{45}$ is accompanied by an additional doublet $h_{45}^{\prime}$ and $\Phi_{45}$ by $\Phi_{45}^{\prime}$, and the color triplets $\left(D_{45}, \bar{D}_{45}\right)$ are absent. These are listed in tables 4,5 and 6 .

While the subgroup $S U(3)_{C} \times S U(2)_{L} \times U(1)_{B-L} \times U(1)_{T_{3_{R}}}$ of $S O(10)$ treats all three families universally, it is easy to see from table 1 that the pairs $\left(U_{1}, U_{4}\right),\left(U_{2}, U_{5}\right)$ and $\left(U_{3}, U_{6}\right)$, respectively couple to families 1,2 and 3 in an identical fashion. Thus, on the one hand, these six $U(1)$ symmetries, having their origin in $S O(44)$, distinguish between the three families, unlike a GUT symmetry like $S O(10)$; thereby they serve as the origin of flavor symmetries, which are needed to explain the hierarchical Yukawa couplings of the three families (see below). On the other hand, as stated before, they preserve the full permutation symmetry with respect to the three families.

It is easy to check that solution I (ref. [13) contains six anomalous $U(1)$ symmetries: $\operatorname{Tr} U_{1}=\operatorname{Tr} U_{2}=\operatorname{Tr} U_{3}=24, \operatorname{Tr} U_{4}=\operatorname{Tr} U_{5}=\operatorname{Tr} U_{6}=-12$. These can be expressed by one anomalous combination which is unique and five non-anomalous 




$$
U_{A}=\frac{1}{\sqrt{15}}\left(2\left(U_{1}+U_{2}+U_{3}\right)-\left(U_{4}+U_{5}+U_{6}\right)\right) ; \operatorname{Tr} Q_{A}=\frac{1}{\sqrt{15}} 180 .
$$

One choice for the five anomaly-free combinations is given by

$$
\begin{aligned}
U_{12} & =\frac{1}{\sqrt{2}}\left(U_{1}-U_{2}\right) \quad, \quad U_{\psi}=\frac{1}{\sqrt{6}}\left(U_{1}+U_{2}-2 U_{3}\right), \\
U_{45} & =\frac{1}{\sqrt{2}}\left(U_{4}-U_{5}\right), \quad U_{\zeta}=\frac{1}{\sqrt{6}}\left(U_{4}+U_{5}-2 U_{6}\right), \\
U_{\chi} & =\frac{1}{\sqrt{15}}\left(U_{1}+U_{2}+U_{3}+2 U_{4}+2 U_{5}+2 U_{6}\right) .
\end{aligned}
$$

Note that the anomalous $U(1)$, containing the sums of $U_{1,2,3}$ and $U_{4,5,6}$ is universal with respect to all three families. This flavor universality of the anomalous $U(1)$ is thus a consequence of the family permutation symmetry of the six $U(1)$-interactions, mentioned above. Of the anomaly free combinations $U_{12}, U_{\psi}, U_{45}$, and $U_{\zeta}$ are clearly family dependent, but $U_{\chi}$ is family universal.

It is worth noting that while solution II (ref. [14) differs in detail from solution I as regards its spectrum of Higgs multiplets and the $S O(10)$ singlets, its gauge interactions nevertheless possess the full permutation symmetry with respect to the three families just like solution I. In this case, however, there are only three anomalous symmetries $U_{1,2,3}$, which can be expressed by one anomalous and two anomaly free combinations :

$$
\begin{aligned}
& U_{A}=\frac{1}{\sqrt{3}}\left(U_{1}+U_{2}+U_{3}\right) ; \quad \operatorname{TrQ} \mathrm{A}=\frac{1}{\sqrt{3}} 72 \\
& U_{12}=\frac{1}{\sqrt{2}}\left(U_{1}-U_{2}\right) \quad ; \quad U_{\psi}=\frac{1}{\sqrt{6}}\left(U_{1}+U_{2}-2 U_{3}\right)
\end{aligned}
$$

Note that the anomalous $U(1)$ is again family-universal, though $U_{12}$ and $U_{\psi}$ are not.

We next examine the superpotential and the issue of generating relevant mass terms which would trigger SUSY-breaking in the string solution of ref. 13.

* The normalization of the different $U(1)$ combinations is fixed by the requirement that the conformal dimension of the massless states still gives $\bar{h}=1$ in the new basis. We remark in advance that the proper normalization must be taken as it affects the minimization of the potential (see below). 


\section{Superpotential and SUSY breaking}

The relevant terms in the cubic level superpotential of solutions I and II are given by

$$
\begin{aligned}
W & =\left[u_{L_{1}}^{c} Q_{1} \bar{h}_{1}+N_{L_{1}}^{c} L_{1} \bar{h}_{1}+u_{L_{2}}^{c} Q_{2} \bar{h}_{2}+N_{L_{2}}^{c} L_{2} \bar{h}_{2}+u_{L_{3}}^{c} Q_{3} \bar{h}_{3}+N_{L_{3}}^{c} L_{3} \bar{h}_{3}\right] \\
& +\left[h_{1} \bar{h}_{2} \bar{\Phi}_{12}+h_{1} \bar{h}_{3} \bar{\Phi}_{13}+h_{2} \bar{h}_{3} \bar{\Phi}_{23}+\bar{h}_{1} h_{2} \Phi_{12}+\bar{h}_{1} h_{3} \Phi_{13}+\bar{h}_{2} h_{3} \Phi_{23}\right]+ \\
& +h_{3} \bar{h}_{45} \Phi_{45}+\bar{h}_{3} h_{45} \bar{\Phi}_{45}+\left(\Phi_{23} \bar{\Phi}_{13} \Phi_{12}+\bar{\Phi}_{23} \Phi_{13} \bar{\Phi}_{12}\right)+\ldots
\end{aligned}
$$

Here a common normalization constant $\sqrt{2} g$ is not exhibited. Note that the Yukawa couplings given in the first square bracket and effectively the second bracket as well respect the family permutation symmetry, which simultaneously permutes the three families and the Higgs-multiplets $\left(\bar{h}_{1}, \bar{h}_{2}, \bar{h}_{3}\right)$, and likewise the $\Phi_{i j}$ 's, but the rest of the superpotential (including higher order terms), which is not shown, does not.

Note that owing to the constraints of the flavor $U(1)_{i}$-symmetries, which distinguish between the families, and the Higgs multiplets, $\bar{h}_{1}$ couples at the cubic level of the superpotential only to family 1 and not to families 2 and 3; Similarly $\bar{h}_{2}$ and $\bar{h}_{3}$ couple only to families 2 and 3 respectively. Now, for the case of solution I (ref. [13]), where contributions of higher dimensional operators to the Higgs mass matrix have been analyzed in detail [26], it has been shown that the pair $h_{3}$ and $\bar{h}_{3}$ necessarily become superheavy since their masses receive contributions from the cubic level superpotential terms; and only one pair of doublets - i.e. either $\left(\bar{h}_{1}, h_{45}\right)$ or $\left(\bar{h}_{2}, h_{45}\right)$ - remains light, while the remaining pairs become medium heavy $\left(\sim 10^{12} \mathrm{GeV}\right)$. It is easy to verify that for solution II (ref. [14]) as well, $h_{3}$ and $\bar{h}_{3}$ become superheavy [27]. The mass pattern of the remaining Higgs doublets depend on the structure of the higher dimensional operators and the allowed pattern of VEVs. Considering the similarity of the massless spectrum in the observable sector for the two cases, however, it seems rather plausible that the Higgs spectrum for the two cases would be quite similar. Following Ref. [14, 27] we will proceed by assuming that only one

pair of Higgs doublets, like $\left(\bar{h}_{1}, h_{45}\right)$ or $\left(\bar{h}_{2}, h_{45}\right)$, remain light for both solutions I and II, and that the remaining pairs become medium or superheavy. 
Since only the light Higgs scalars acquire VEVs (radiatively), it would follow, for the Higgs spectrum mentioned above, that the up-quark member of only one family i.e. the top and $\nu_{\tau}$ would get masses at the level of cubic terms in $W$. The masses of the other quarks and their mixings would arise through successively higher dimensional operators, which permit their couplings to the light Higgs-pair. Thus, a hierarchy in fermion masses and mixings arises in spite of the permutation symmetry of the cubic Yukawa couplings (see ref. [13] and especially ref. [14] for details of this discussion). Thus, ultimately, such a hierarchy has its origin in two features : (a) the family dependent $U(1)_{i}$-symmetries, which force the three families to have Yukawa couplings with three distinct Higgs-multiplets, and (b) the spontaneously generated asymmetric Higgs mass-matrix.

We now turn to the pattern of symmetry breaking below the string-scale. The anomalous $U(1)_{A}$ is broken by the Dine-Seiberg-Witten mechanism 28 in which a potentially large Fayet-Iliopoulos $D$-term $\xi$ is generated by the VEV of the dilaton field. Such a $D$-term would, in general, break supersymmetry, unless there is a direction in the scalar potential $\hat{\phi}=\sum \alpha_{i} \phi_{i}$ which is $F$-flat and also $D$-flat with respect to all the non-anomalous gauge symmetries and in which $\sum Q_{A}^{i}\left|\alpha_{i}\right|^{2}<0$. If such a direction exists, it will acquire a VEV, canceling the Fayet-Iliopoulos $\xi$-term, restoring supersymmetry and stabilizing the vacuum. The exception to this picture arises if there exist mass terms $(m)$ for certain relevant fields carrying anomalous charge; in this case the anomalous $D$-term and the $F$-terms would necessarily acquire nonvanishing VEVs that are proportional to $m$ and SUSY would be broken.

The set of $D$ and $F$ flat constraints, in the absence of such mass terms, is given by,

$$
\begin{aligned}
& \left\langle D_{A}\right\rangle=\left\langle D_{\alpha}\right\rangle=\left\langle D_{\beta}\right\rangle=\left\langle F_{i} \equiv \frac{\partial W}{\partial \eta_{i}}\right\rangle=0 \\
& D_{A}=\left[K_{A}+\sum Q_{A}^{k}\left|\chi_{k}\right|^{2}+\xi\right] \\
& D_{\alpha}=\left[K_{\alpha}+\sum Q_{\alpha}^{k}\left|\chi_{k}\right|^{2}\right], \alpha \neq A \\
& \xi=\frac{g^{2}\left(\operatorname{Tr} Q_{A}\right)}{192 \pi^{2}} M_{\mathrm{Pl}}^{2}
\end{aligned}
$$


Here $\chi_{k}$ are the fields which acquire VEVs of order $\sqrt{\xi}$, while the $K$-terms contain fields $\eta_{i}$ like squarks, sleptons and Higgs bosons whose VEVs vanish, at this scale. $Q_{A}^{k}$ and $Q_{A}^{i}$ denote the anomalous and non-anomalous charges, which are listed in eqs. (2.2)-(2.7) for solutions I and II, and $M_{\mathrm{Pl}} \approx 2 \times 10^{18} \mathrm{GeV}$ denotes the reduced Planck mass. The solution (i.e. the choice of fields with non-vanishing VEVs) to the set of equations (3.2)-(3.4), though nontrivial, is not unique. A few alternative solutions have been considered in refs. [13, 14, 26, 19].

As a general guide, note that $\xi$ is positive and is of order $10^{-2} M_{\mathrm{Pl}}^{2}$. To cancel the $\xi$-term in $\left\langle D_{A}\right\rangle$, in the absence of mass terms, at least one field with negative $Q_{A}$ must acquire a VEV. A large set of solutions including those of refs. [13] and [14] assigns nonzero VEV to $\Phi_{45}$, which is the field with the largest negative $Q_{A}$. If $\Phi_{45}$ (or a suitable alternative), acquiring a VEV, is charged with respect to one of the other symmetries, some additional fields must also acquire VEVs, so that the full set of $\left\langle D_{A, \alpha, j}\right\rangle$ must vanish.

To demonstrate how SUSY breaking could arise it is instructive to consider first a simple pattern of VEVs satisfying eqs. (3.2)-(3.4), for the case of solution II (ref. [14]). We will subsequently study a more complicated pattern of VEVs for solution I (ref. [13]).

\section{SUSY breaking in solution II:}

As an instructive example we consider a pattern which assigns nonzero VEVs of order $\sqrt{\xi}$ to only two fields

$$
\left\langle\left\{\Phi_{45}, \Phi_{45}^{\prime}\right\}\right\rangle \neq 0
$$

All other fields have zero VEV. The charges $\left(Q_{A}, Q_{\psi}, Q_{12}\right)$ for $\Phi_{45}$ are $(-2,+1,0)$, and those for $\Phi_{45}^{\prime}$ are $(0,-3,0)$ (see eqs. (2.7) and table II). Their contributions to the respective $D$-terms are thus given by,

$$
\begin{aligned}
D_{A} & =\frac{1}{\sqrt{3}}\left[K_{A}-2\left(\left|\Phi_{45}\right|^{2}-\left|\bar{\Phi}_{45}\right|^{2}\right)+\hat{\xi}\right] \\
D_{\psi} & =\frac{1}{\sqrt{6}}\left[K_{\psi}+\left(\left|\Phi_{45}\right|^{2}-\left|\bar{\Phi}_{45}\right|^{2}\right)-3\left|\Phi_{45}\right|^{2}\right] \\
D_{12} & =\frac{1}{\sqrt{2}} K_{12}
\end{aligned}
$$


where $\hat{\xi}=\sqrt{3} \xi$. The contribution of $\Phi_{45}$ and $\Phi_{45}^{\prime}$ to all other $D$-terms are zero. The $K$-terms contain fields like squarks, sleptons and Higgs bosons which have zero VEVs. Although $\bar{\Phi}_{45}$ is assigned zero VEV, its contribution is still exhibited to demonstrate that it would be forced to have zero VEV in the presence of a mass term. All the F and D flat conditions are satisfied at the cubic level of the superpotential by assigning

$$
\left|\left\langle\Phi_{45}\right\rangle_{0}\right|^{2}=3\left|\left\langle\Phi_{45}^{\prime}\right\rangle_{0}\right|^{2}=\hat{\xi} / 2
$$

All other VEVs are zero. The subscript zero signifies that the VEVs are obtained in the zero mass limit. Now introduce an effective mass-term $m\left(<<M_{\text {string }}\right)$ for $\Phi_{45}$ and $\bar{\Phi}_{45}$ in the superpotential :

$$
W \supset m \Phi_{45} \bar{\Phi}_{45}
$$

We will discuss how such a mass term is likely to arise through higher dimensional operators in string theory. The effective potential then takes the form:

$$
V=\frac{g^{2}}{2}\left(D_{A}^{2}+D_{\psi}^{2}+D_{12}^{2}\right)+m^{2}\left(\left|\Phi_{45}\right|^{2}+\left|\bar{\Phi}_{45}\right|^{2}\right)
$$

For simplicity of writing, we have put just one gauge coupling; in practice the various gauge couplings would differ due to the running even if they are equal at the string scale. It is now easy to verify that minimization of the potential would lead to a shift in the VEVs of $\Phi_{45}$ and $\Phi_{45}^{\prime}$.

The extremum conditions lead to the following constraints:

$$
\begin{aligned}
& \frac{\partial V}{\partial \Phi_{45}}=0 \Rightarrow\left(\Phi_{45}\right)^{\dagger}\left[-2 D_{A}+\frac{D_{\psi}}{\sqrt{2}}+\sqrt{3} \frac{m^{2}}{g^{2}}\right]=0 \\
& \frac{\partial V}{\partial \Phi_{45}^{\prime}}=0 \Rightarrow\left(\Phi_{45}^{\prime}\right)^{\dagger}\left[D_{\psi}\right]=0 \\
& \frac{\partial V}{\partial \bar{\Phi}_{45}}=0 \Rightarrow\left(\bar{\Phi}_{45}\right)^{\dagger}\left[2 D_{A}-\frac{D_{\psi}}{\sqrt{2}}+\sqrt{3} \frac{m^{2}}{g^{2}}\right]=0
\end{aligned}
$$

Here the fields and the $D$-terms to the right of the arrows stand for the VEVs of the respective entities. Since $\left\langle\Phi_{45}\right\rangle \neq 0$, eq. (3.13) clearly shows that $\left\langle D_{A}\right\rangle$ and/or $\left\langle D_{\psi}\right\rangle$ must be of order $m^{2} / g^{2}$ and thus SUSY is broken. Since $\left\langle\Phi_{45}^{\prime}\right\rangle \neq 0$, eq. (3.14) 
implies $\left\langle D_{\psi}\right\rangle=0$. Eq. (3.13) then yields $\left\langle D_{A}\right\rangle=\left(\sqrt{3} m^{2}\right) /\left(2 g^{2}\right)$. Substituting this in Eq. (3.15) we see that $\left\langle\bar{\Phi}_{45}\right\rangle$ must remain zero, even with $m \neq 0$. Now, using the expressions for $D_{A}$ and $D_{\psi}$ given in Eqs. (3.7) and (3.8), we can determine the VEVs of $\Phi_{45}$ and $\Phi_{45}^{\prime}$. Thus, we see that, for the special choice of VEVs given by Eq. (3.10), which provides a solution to the $F$ and $D$-flat conditions (eqs. (3.2 3.5)) in the massless limit, the extremum condition lead to a unique solution for the pattern of VEVs in the case of finite mass $\left(m\left(\Phi_{45}\right)=m\left(\bar{\Phi}_{45}\right) \neq 0\right)$ :

$$
\begin{aligned}
\left|\left\langle\Phi_{45}\right\rangle\right|^{2} & =\frac{\hat{\xi}}{2}-\frac{3}{4} \frac{m^{2}}{g^{2}} ; \\
\left|\left\langle\Phi_{45}^{\prime}\right\rangle\right|^{2} & =\frac{\hat{\xi}}{6}-\frac{1}{4} \frac{m^{2}}{g^{2}} ; \\
\left|\left\langle\bar{\Phi}_{45}\right\rangle\right|^{2} & =0 \\
\left\langle D_{A}\right\rangle & =\frac{\sqrt{3}}{2} \frac{m^{2}}{g^{2}} ; \\
\left\langle D_{\psi}\right\rangle & =\left\langle D_{12}\right\rangle=0 \\
F\left(\Phi_{45}\right) & =F\left(\Phi_{45}^{\prime}\right)=0 ; \\
F\left(\bar{\Phi}_{45}\right) & =m \sqrt{\frac{\hat{\xi}}{2}-\frac{3}{4} \frac{m^{2}}{g^{2}}}
\end{aligned}
$$

It may be verified that this $\mathrm{VEV}$-pattern in fact minimizes the potential. Note that for this simple example, the $D$-term of only the anomalous charge $Q_{A}$, which is family universal, is non-zero, but those of the non-universal charges $Q_{\psi}$ and $Q_{12}$ vanish owing to minimization. This special feature arises because there are only two fields $\left(\Phi_{45}\right.$ and $\left.\Phi_{45}^{\prime}\right)$, having non-zero $\mathrm{VEVs}$, and they contribute only to two $D$ terms $\left(D_{A}\right.$ and $\left.D_{\psi}\right)$, but not to $D_{12}$. Thus, to start with, $D_{12}=0$. Furthermore, $\Phi_{45}^{\prime}$ contributes only to $D_{\psi}$, but not to $D_{A}$. Thus, extremization of $\mathrm{V}$ with respect to $\Phi_{45}^{\prime}$ forces $D_{\psi}=0$ as well (see eq. (3.14)).

Vanishing of the non-universal $D$-terms has the desirable consequence that squarks of all three families receive the same contribution to their masses from the $D$-terms, in spite of the presence of the non-universal flavor symmetries:

$$
\left[m_{\tilde{q}_{i}}^{2}\right]_{D_{A}}=g^{2} Q_{A}^{i}\left\langle D_{A}\right\rangle=Q_{A}^{i}\left(\frac{\sqrt{3}}{2} m^{2}\right)=\frac{m^{2}}{4}
$$


Likewise for the sleptons. Here $Q_{A}^{i}$ denotes the anomalous charge of the respective field. For solution II, $Q_{A}^{i}=\left(Q_{1}+Q_{2}+Q_{3}\right)^{i}$ is not only family-universal, but it is also positive and the same $(=1 / \sqrt{12})$ for all members of a family. Thus, $\tilde{Q}_{L}, \tilde{d}_{R}, \tilde{L}$, $\tilde{u}_{R}$ and $\tilde{e}_{R}$ are degenerate (barring small $F$-term contributions) at the scale of $\sqrt{\xi}$. We will return to this point in section 5 .

It has been suggested in ref. [5], that in a supergravity theory the squarks and the sleptons are expected to receive contributions to their masses from the Kähler potential through $F$-terms like $\lambda \int d^{4} \theta\left(\bar{\Phi}_{45}\right)\left(\bar{\Phi}_{45}\right)^{\dagger} q_{i} q_{i}^{\dagger} / M_{\mathrm{Pl}}^{2}$, where $\lambda=\mathcal{O}(1)$. Although these operators conserve all gauge symmetries, in a string theory one still needs to ascertain whether they satisfy the string-selection rules; otherwise $\lambda$ would be small $(\leq 1 / 10)$ compared to unity. Deferring the study of this issue to a later work, we note that the contribution of these terms, if they are present, to squark masses are given by

$$
\left[\Delta m_{\tilde{q}_{i}}^{2}\right]_{F} \approx \frac{\lambda\left|\left\langle F\left(\bar{\Phi}_{45}\right)\right\rangle\right|^{2}}{M_{P l}^{2}} \approx \frac{\lambda m^{2} \hat{\xi}}{2 M_{P l}^{2}}=\lambda m^{2} \epsilon / 2
$$

where $\epsilon=\hat{\xi} / M_{p l}^{2}$. With $\hat{\xi}=\sqrt{3} \xi$, and $\xi$ given by (3.5) and $\operatorname{Tr} Q_{A}=72 / \sqrt{3}$ [14], we expect $\epsilon \approx 1 / 60$. In general, the $F$-term contributions are not expected to be universal, unless the Kähler potential possesses a certain symmetry (see remarks below). Even then, and even if $\lambda \approx(1 / 2-1)$ (say), these $F$-term contributions are suppressed compared to the $D$-term contribution (eq. (3.19)) by about a factor of (60-30), for $\epsilon \approx 1 / 60$. Degeneracy to this extent suffices to account for the smallness of at least the real part of the $K^{0}-\bar{K}^{0}$ transition amplitude, if $m_{\tilde{d}} \approx$ $m_{\tilde{s}} \geq(700-1400) \mathrm{GeV}$ [29]. Understanding the extreme smallness of the imaginary part of the $K^{0}-\bar{K}^{0}$ amplitude, which we do not address here would need additional considerations, based perhaps on symmetry properties, which may explain why the relevant phase angle is so small $\leq 10^{-2}$ !

${ }^{*}$ In quoting lower limits on squark-masses, we have used a value for the product of mixing angles $\left(\cos \theta_{d}\right)\left(\sin \theta_{d}\right) \approx(1 / 8-1 / 10)$, for the down quark-sector, which seems reasonable.

${ }^{\dagger}$ The problem of SUSY CP-violation, in the context of models of SUSY-breaking as proposed here, is discussed in a forthcoming paper by K.S. Babu and J.C. Pati, where a natural explanation for the extreme smallness of the $\epsilon-$ parameter is given. 
At this stage, the following property of the string solutions under study is worth noting. We have observed that the family permutation symmetry is exact at the level of the NAHE set in that it holds for the gauge interactions as well as for the super and Kähler potentials. Even after the introduction of the additional boundary condition basis vectors $(\alpha, \beta, \gamma)$ the permutation symmetry is still retained in the gauge interactions, as well as in the cubic Yukawa interactions of the quarks and the leptons with the Higgs fields and in the $h_{i} \bar{h}_{j} \bar{\Phi}_{i j}$-terms of the superpotential $W$ (see eq. (3.1)). It is lost in $W$ only through (a) $\mathcal{O}\left(\Phi^{3}\right)$-terms, (b) terms involving Higgs and the exotic fields but not quarks and leptons (these are not shown in (3.1)), and (c) possibly some higher dimensional terms. As a result, the effect of this loss of the permutation symmetry on the quarks and leptons and their superpartners is extremely mild in that it is felt by them only through at most two-loop effects and higher dimensional terms (whose contributions to the masses of the $(d, s)$-squarks are less than or of order $200 \mathrm{MeV}$ ). If the Kähler potential retains the family permutation symmetry to the same extent as the superpotential $W$, which is plausible, but which is an issue that needs to be examined, even the $F$-term contributions given by (3.20) would be very nearly family universal. In this case, and/or if $\lambda \leq 1 / 10$, the degree of squark-degeneracy would be far better (in this case, one would have $\left.\left[\left(\tilde{m}_{i}^{2}-\tilde{m}_{j}^{2}\right) / \tilde{m}^{2}\right]<<10^{-2}\right)$ than that indicated above. We defer the study of the Kähler potential to later work. For the present, we will proceed by taking the squark degeneracy ratio to be no better than 1/30 - 1/60, as obtained above.

The gauginos of the Standard Model gauge sector (i.e. gluinos, winos etc.) could, in general, receive masses through operators of the form: $\lambda^{\prime} \int d^{2} \theta \Phi_{45} \bar{\Phi}_{45} W_{a} W_{a} / M_{P l}^{2}$ $(a=1,2,3)[5]$ which yields:

$$
m\left(\lambda_{a}\right) \approx \lambda^{\prime}\left\langle F\left(\bar{\Phi}_{45}\right)\left\langle\Phi_{45}\right\rangle / M_{P l}^{2} \approx \lambda^{\prime} \epsilon m\right.
$$

where $\lambda^{\prime} \leq \mathcal{O}(1)$. We see the hierarchy

$$
\left[m^{2}\left(\tilde{q}_{i}\right) \approx Q_{A}^{i}\left(\sqrt{3} m^{2} / 2\right)\right]>\left[\Delta m_{\tilde{q}_{i}}^{2} \approx \lambda \epsilon\left(m^{2} / 2\right)\right]>\left[m_{\lambda_{a}}^{2} \approx \lambda^{\prime} \epsilon^{2} m^{2}\right]
$$

Because of this hierarchy, it is clear that if SUSY breaking proceeds entirely through anomalous $U(1)$, the gluinos typically would be rather light. From (3.21), 
one obtains: $m_{\tilde{g}} \approx 2 \lambda^{\prime} \epsilon m_{\tilde{q}} \approx \lambda^{\prime}(20-60) \mathrm{GeV}$, for $m_{\tilde{q}} \approx(1-3) \mathrm{TeV}$; this may be too light, compared to the observed limit on $m_{\tilde{g}}$ of $130 \mathrm{GeV}$, unless $\lambda^{\prime} \geq 2$ and $m_{\tilde{q}} \geq 3 \mathrm{TeV}$. To make matters worse, for string solutions, as considered here, $\lambda^{\prime}$ vanishes at tree level and can only arise through quantum loops; thus it is expected to be small. This suggests that SUSY breaking through anomalous $U(1)$, quite plausibly, is accompanied by an additional source which provides the dominant contribution to gluino masses $(\sim(1-$ few $)(100 \mathrm{GeV}))$, while preserving the squark-degeneracy, obtained through $U(1)_{A}$. We comment on this possibility in section 6 .

We should note that, for the sake of convenience, we have evaluated the VEVs of $\Phi_{45}, \bar{\Phi}_{45}$, and $\bar{\Phi}_{45}^{\prime}$ and the auxiliary fields in the flat limit. It has, however, been shown in Ref. [5], that the inclusion of supergravity effects do not restore supersymmetry, though they shift the VEVs of fields; e.g. $\left\langle\bar{\Phi}_{45}\right\rangle$ acquires a non-zero value which is typically bounded above by $\left\langle\Phi_{45}\right\rangle$. Such shifts, however, do not alter the pattern of soft masses and the hierarchy shown in (3.22).

Before discussing the origin of the mass term $m$ and certain phenomenological issues, we first discuss SUSY-breaking in solution I ([13).

\section{SUSY breaking in solution I:}

This case is more complex than the one presented above, because it has six $U(1)^{\prime} s$ (in contrast to three relevant ones for solution II), four of which are non-universal, and typically several fields (not merely two) must acquire VEVs of order $\sqrt{\xi}$ to satisfy the $F$ and $D$ flat conditions. The instructive example presented above prompts us nevertheless to ask: (i) can one still find at least a local minimum of the potential $\mathrm{V}$ which leads to nonzero VEVs for the $D$-terms of only the family universal charges i.e. $Q_{A}$ and $Q_{\chi}$ ? (ii) If so, is that a global minimum ? We find that the answer to the first question, interestingly enough is in the affirmative, and that to the second, though hard to assess in general, is also found to be the same for the limited subset of field-space, considered here.

Consider now a solution to the $D$ and $F$ flat conditions (eqs. 3.2 3.5) for the case of solution I (ref. [13]), which assigns non-zero VEVs of order $\sqrt{\xi}$ to the following 
set of fields:

$$
\left.\left\{\Phi_{45}, \bar{\Phi}_{13}, \bar{\Phi}_{3}^{-}, \bar{\Phi}_{1}^{+}, \bar{\Phi}_{2}^{-}, \xi_{1}\right\}\right\rangle=\mathcal{O}(\sqrt{\xi})
$$

All other fields have zero VEVs at the scale $\sqrt{\xi}$.

The contributions of these fields to the $D$-terms of the symmetries listed in eqs (2.2) and (2.3) are given by (compare with eqs. (3.7 3.9)).

$$
\begin{aligned}
D_{A} & =\frac{1}{\sqrt{15}}\left[K_{A}-\sigma^{2}+4\left(\left|\bar{\Phi}_{45}\right|^{2}-\left|\Phi_{45}\right|^{2}\right)+\hat{\xi}\right] \\
D_{\psi} & =\frac{1}{\sqrt{6}}\left[K_{\psi}-3\left|\bar{\Phi}_{13}\right|^{2}+\left(\left|\Phi_{45}\right|^{2}-\left|\bar{\Phi}_{45}\right|^{2}\right)\right] \\
D_{12} & =\frac{1}{\sqrt{2}}\left[K_{12}-\left|\bar{\Phi}_{13}\right|^{2}-2\left|\bar{\Phi}_{12}\right|^{2}+\frac{\sigma^{2}}{3}-\frac{\beta^{2}}{3}-\delta^{2}\right] \\
D_{45} & =\frac{1}{\sqrt{2}}\left[K_{45}+|\delta|^{2}\right] \\
D_{\zeta} & =\frac{1}{\sqrt{6}}\left[K_{\zeta}+\beta^{2}\right] \\
D_{\chi} & =\frac{1}{\sqrt{15}}\left[K_{\chi}+2 \sigma^{2}+2\left(\left|\bar{\Phi}_{45}\right|^{2}-\left|\Phi_{45}\right|^{2}\right)\right]
\end{aligned}
$$

Here $\hat{\xi} \equiv \sqrt{15} \xi$. As before, the $\mathrm{K}$-terms contain fields like squarks, sleptons and Higgs-bosons which have zero VEVs. Anticipating that a field like $\bar{\Phi}_{12}$ (or $\Phi_{23}$ ) which is charged under $U_{12}$ and possibly $U_{\psi}$, but not the other $U(1)^{\prime} s$, may need to acquire a VEV of order $m<<\sqrt{\xi}$, in the presence of a mass-term $m$, we have exhibited its contribution. The combinations $\sigma, \beta$ and $\delta$ are defined by:

$$
\begin{aligned}
& \sigma^{2} \equiv\left|\Phi_{1}^{+}\right|^{2}+\left|\bar{\Phi}_{2}^{-}\right|^{2}+\left|\bar{\Phi}_{3}^{-}\right|^{2} \\
& \beta^{2} \equiv\left|\Phi_{1}^{+}\right|^{2}+\left|\bar{\Phi}_{2}^{-}\right|^{2}-2\left|\bar{\Phi}_{3}^{-}\right|^{2} \\
& \delta^{2} \equiv\left|\Phi_{1}^{+}\right|^{2}-\left|\bar{\Phi}_{2}^{-}\right|^{2}
\end{aligned}
$$

It may be verified that all the $F$ - and $D$-flat constraints $\left(F_{i}=D_{A}=D_{\alpha}=0\right)$ are satisfied for the $m=0$ cubic-level superpotential, with $\left|\left\langle\Phi_{45}\right\rangle_{0}\right|^{2}=\hat{\xi} / 5$, and $\begin{aligned} &\left|\left\langle\bar{\Phi}_{13}\right\rangle_{0}\right|^{2}=\left|\left\langle\Phi_{1}^{+}\right\rangle_{0}\right|^{2}=\left|\left\langle\bar{\Phi}_{2}^{-}\right\rangle_{0}\right|^{2}=\left|\left\langle\bar{\Phi}_{3}^{-}\right\rangle_{0}\right|^{2}=\hat{\xi} / 15, \text { i.e. } \\ &\left|\left\langle\Phi_{45}\right\rangle_{0}\right|^{2}=\left|\langle\sigma\rangle_{0}\right|^{2}=3\left|\left\langle\bar{\Phi}_{13}\right\rangle_{0}\right|^{2}=\frac{\hat{\xi}}{5} \\ &\left\langle\beta^{2}\right\rangle_{0}=\left\langle\delta^{2}\right\rangle_{0}=\left\langle\bar{\Phi}_{12}\right\rangle_{0}=0\end{aligned}$ 
All other VEVs are zero. As before, the subscript zero signifies that the VEVs are obtained in the limit of zero-mass for all the fields. The VEV of the singlet $\xi_{1}$ is not determined by the $F$ and $D$ flat constraints (at least at the cubic level superpotential). Independent phenomenological considerations including quark-lepton masses and mixings, however imply that the $\operatorname{VEV}\left\langle\xi_{1}\right\rangle$ must be of order $\sqrt{\xi} \sim \mathcal{O}\left(g^{2} M_{s t} / 4 \pi\right)$ [26]. In the absence of a complete solution to the vacuum selection in string theory, we will proceed by imposing this choice.

Allowing for a mass term $m$ as in (3.11), and extremizing the potential

$$
V=\frac{g^{2}}{2} \sum_{\alpha} D_{\alpha}^{2}+m^{2}\left(\left|\Phi_{45}\right|^{2}+\left|\bar{\Phi}_{45}\right|^{2}\right)
$$

with respect to $\Phi_{45}, \bar{\Phi}_{13}, \sigma$ and $\bar{\Phi}_{12}$, respectively we obtain (compare with eqs. (3.13)(3.15)):

$$
\begin{aligned}
\frac{\partial V}{\partial \Phi_{45}} & =0 \Rightarrow\left(\Phi_{45}\right)^{\dagger}\left[4\left(D_{A}+\frac{D_{\chi}}{2}-\frac{\sqrt{15}}{4} m^{\prime^{2}}\right)-\sqrt{\frac{5}{2}} D_{\psi}\right]=0 \\
\frac{\partial V}{\partial \bar{\Phi}_{13}} & =0 \Rightarrow\left(\bar{\Phi}_{13}\right)^{\dagger}\left[D_{12}+\sqrt{3} D_{\psi}\right]=0 \\
\frac{\partial V}{\partial \sigma} & =0 \Rightarrow \sigma\left[\left(D_{A}-2 D_{\chi}\right)-\sqrt{\frac{5}{6}} D_{12}\right]=0 \\
\frac{\partial V}{\partial \bar{\Phi}_{12}} & =0 \Rightarrow\left(\bar{\Phi}_{12}\right)^{\dagger}\left(D_{12}\right)=0
\end{aligned}
$$

Here $m^{\prime 2} \equiv m^{2} / g^{2}$. variations with respect to $\beta$ and $\delta$ are not exhibited because these can be satisfied consistently by preserving their zero mass values: $\beta=\beta_{0}=0$ and $\delta=\delta_{0}=0$. Thus, from eqs. (3.27) and (3.28), $D_{45}=D_{\zeta}=0$. As in the previous example, we see from eq. (3.36) that $\left\langle D_{A}\right\rangle,\left\langle D_{\chi}\right\rangle$ and/or $\left\langle D_{\psi}\right\rangle$ must be of order $\left(m^{2}\right)$, and thus SUSY is broken.

Unlike the previous example, however, where $\Phi_{45}^{\prime} \neq 0$ uniquely led (via eq. (3.10)) to $D_{\psi}=0$, we see that eq. (3.37), can be satisfied, given $\bar{\Phi}_{13} \neq 0$, by choosing either (a) $D_{12}=D_{\psi}=0$, or (b) $D_{12}=-\sqrt{3} D_{\psi}=\mathcal{O}\left(m^{2}\right) \neq 0$. In short, the solution for the $D$ 's do not appear to be unique. Case (a) would, of course be phenomenologically preferable, because it would lead to family universal squark masses. We consider these two cases by turn and show that minimization of the potential in fact favors case (a) over case (b). 
Case (a) $D_{12}=D_{\psi}=0$

Given $D_{12}=0$, eq. (3.39) can be satisfied by choosing either $\bar{\Phi}_{12}=0$ or $\bar{\Phi}_{12}=$ $\mathcal{O}(m) \neq 0$. We will see that internal consistency will fix $\bar{\Phi}_{12}=\mathcal{O}(m) \neq 0$, if $D_{12}=0$.

Given $D_{12}=D_{\psi}=0$, eqs. (3.36) and (3.38) imply: (i) $D_{A}+D_{\chi} / 2=\sqrt{15} m^{\prime^{2}} / 4$ and (ii) $D_{A}=2 D_{\chi}$, which in turn imply:

$$
D_{A}=\sqrt{\frac{3}{5}} m^{\prime^{2}} \quad ; \quad D_{\chi}=\sqrt{\frac{3}{5}} \frac{m^{\prime^{2}}}{2},
$$

Now, given $\beta=\delta=0$, looking at the compositions of the $D$-terms (eqs. (3.25 3.29), $D_{\psi}=0$ and $D_{12}=0$ imply:

$$
\begin{aligned}
\left|\bar{\Phi}_{13}\right|^{2} & =\left|\Phi_{45}\right|^{2} / 3 \\
\sigma^{2} & =\left|\Phi_{45}\right|^{2}+6\left|\bar{\Phi}_{12}\right|^{2}
\end{aligned}
$$

Substituting (3.42) in $D_{\chi}$ (see eq. (3.29)), and putting $D_{\chi}=\sqrt{3 / 5}\left({m^{\prime}}^{2} / 2\right)$ (see (3.40)), we get

$$
\left|\bar{\Phi}_{12}\right|^{2}=\frac{m^{\prime^{2}}}{8}
$$

Thus, internal consistency for case (a) $\left(D_{12}=D_{\psi}=0\right)$ imply that a field like $\bar{\Phi}_{12}$ (alternatively $\Phi_{23}$ will also be adequate), which had a zero VEV to begin with (i.e. for $m=0$ ), must acquire a non-zero VEV of order $m$.

Solving for the nonzero VEVs, and collecting the results, we obtain

$$
\begin{aligned}
& \left|\left\langle\Phi_{45}\right\rangle\right|^{2}=\frac{\hat{\xi}}{5}-\frac{3}{4} m^{\prime^{2}} ;\left|\left\langle\bar{\Phi}_{13}\right\rangle\right|^{2}=\frac{\hat{\xi}}{15}-\frac{m^{\prime^{2}}}{4} ;\left\langle\sigma^{2}\right\rangle=\frac{\hat{\xi}}{5} \\
& \left\langle\bar{\Phi}_{12}\right\rangle^{2}=\frac{m^{\prime^{2}}}{8} ; \\
& \left\langle\bar{\Phi}_{45}\right\rangle^{2}=\left\langle\beta^{2}\right\rangle=\left\langle\delta^{2}\right\rangle=0 \\
& \left\langle D_{A}\right\rangle=\sqrt{\frac{3}{5}} m^{\prime^{2}} ;\left\langle D_{\chi}\right\rangle=\sqrt{\frac{3}{5}} \frac{m^{\prime 2}}{2} ; \\
& D_{\psi}=D_{12}=D_{\zeta}=D_{45}=0 \\
& \left\langle F\left(\Phi_{45}\right)\right\rangle=\left\langle F\left(\bar{\Phi}_{13}\right)\right\rangle=\langle F(\sigma)\rangle=\langle F(\zeta)\rangle=\langle F(\delta)\rangle=0 \\
& \left\langle F\left(\bar{\Phi}_{45}\right)\right\rangle=m \sqrt{\frac{\hat{\xi}}{5}-\frac{3}{4} m^{\prime 2}}
\end{aligned}
$$


It may be verified that the solution presented above is in fact a minimum of $V$. We see that there exists at least a local minimum for which the D-terms of only the universal charges $Q_{A}$ and $Q_{\chi}$ are non-zero. This solution thus has the desirable feature that the $D$-term contributions to the squark-masses, which dominate over $F$ term contributions, are family universal. The rest of the phenomenological discussions (i.e. the gaugino masses and the hierarchy) are qualitatively the same as in solution II (see eqs 3.19 3.22). To be specific, now $\operatorname{Tr} Q_{A}=180 / \sqrt{15}$, but $\hat{\xi}=\sqrt{15} \xi$, so $\epsilon \equiv \hat{\xi} / M_{P l}^{2} \approx 1 / 25$. The contributions of $D_{A}$ and $D_{\chi}$ are given by: $\left[m_{\tilde{d}, \tilde{s}, \tilde{b}}^{2}\right]_{D_{A}, D_{\chi}}=$ $g^{2}\left[Q_{A} D_{\chi}+Q_{\chi} D_{\chi}\right]=m^{2} / 4$, where we have put $\sqrt{15} Q_{A}=3 / 2$ and $\sqrt{15} Q_{\chi}=-1 / 2$ (see table 1 ) and $D_{A}=\sqrt{3 / 5} m^{\prime 2}=2 D_{\chi}$. The $F$-term contributions (see eq. (3.20), which may in general be non-universal, are given by: $\left[m_{\tilde{d}, \tilde{s}, \tilde{b}}^{2}\right]_{F} \approx \lambda\left|F\left(\bar{\Phi}_{45}\right)\right|^{2} / M_{P l}^{2} \approx$ $\lambda\left(m^{2} / 5\right) \epsilon$, which are thus suppressed by about a factor of $(1 / 60-1 / 30)$ f for $\lambda \approx(1 / 2-$ $1)$ ) compared to the universal $D$-term contributions of $g^{2}\left[Q_{A} D_{\chi}+Q_{\chi} D_{\chi}\right]=m^{2} / 4$. As for solution II, this is compatible with the constraints from the real part of the $K^{0}-\bar{K}^{0}$ amplitude, if $m_{\tilde{d}, \tilde{s}} \geq(700-1400) \mathrm{GeV}$.

Case (b): $D_{12}=\mathcal{O}\left(m^{2}\right) \neq 0$

In this case, eqs. (3.39) and (3.37) respectively imply:

$$
\bar{\Phi}_{12}=0, \text { and } D_{\psi}=-D_{12} / \sqrt{3}
$$

substituting (3.51) into (3.36) and using (3.38), we get

$$
D_{A}=\sqrt{\frac{3}{5}} m^{\prime^{2}} \quad ; \quad D_{\chi}=\frac{1}{2}\left(\sqrt{\frac{3}{5}} m^{\prime^{2}}-\sqrt{\frac{5}{6}} D_{12}\right)
$$

Given $\bar{\Phi}_{12}=\beta=\delta=0$, the expressions for $D_{A}, D_{12}$ and $D_{\psi}$ and $D_{\chi}$ given in eqs. (3.24 3.29) respectively yield:

$$
\begin{aligned}
& 4\left|\Phi_{45}\right|^{2}+\sigma^{2}=\hat{\xi}-3 m^{\prime 2} \\
& -\left|\bar{\Phi}_{13}\right|^{2}+\frac{\sigma^{2}}{3}=\sqrt{2} D_{12} \\
& -3\left|\bar{\Phi}_{13}\right|^{2}+\left|\Phi_{45}\right|^{2}=-\sqrt{2} D_{12} \\
& \sigma^{2}-\left|\Phi_{45}\right|^{2}=\frac{3}{4} m^{\prime 2}-\frac{5}{4 \sqrt{2}} D_{12}
\end{aligned}
$$


Combining (3.54) and (3.55), we get

$$
\left|\Phi_{45}\right|^{2}-\sigma^{2}=-4 \sqrt{2} D_{12}
$$

Combining (3.53 3.57), one obtains

$$
\begin{aligned}
& \left|\Phi_{45}\right|^{2}=\frac{\hat{\xi}}{5}-\frac{3}{4} m^{\prime 2}+\frac{1}{4 \sqrt{2}} D_{12} \\
& \sigma^{2}=\frac{\hat{\xi}}{5}-\frac{1}{\sqrt{2}} D_{12} \\
& \left|\bar{\Phi}_{13}\right|^{2}=\frac{\hat{\xi}}{15}-\frac{m^{\prime 2}}{4}+\frac{3}{4 \sqrt{2}} D_{12}
\end{aligned}
$$

Comparing (3.56) and (3.57), we get

$$
D_{12}=\frac{3 \sqrt{2}}{37} m^{\prime 2}
$$

One can verify that the solution for the VEVs presented above again corresponds to a minimum of $V$. To compare the minimum obtained in case (a) with that of case (b) we need to study the variation of $V$ with respect to $D_{12}$. To do so we evaluate the potential at the string unification scale, where the couplings of the $U(1)$ 's are unified.

$$
V=\frac{g^{2}}{2}\left(D_{A}^{2}+D_{\chi}^{2}+D_{\psi}^{2}+D_{12}^{2}\right)+m^{2}\left(\left|\Phi_{45}\right|^{2}+\bar{\Phi}_{45}^{2}\right)
$$

Substituting for $D_{A}, D_{\chi}, D_{\psi}$, and $\left|\Phi_{45}\right|^{2}$ from eqs. (3.52), (3.51), and (3.58), we getf

$$
\frac{V}{\left(g^{2} / 2\right)}=\frac{2}{5} \hat{\xi} m^{\prime^{2}}+\mathcal{O}\left(m^{\prime^{4}}\right)+\frac{13}{24} D_{12}^{2}+{m^{\prime 2}}^{2}\left(-\frac{1}{4 \sqrt{2}}+\frac{1}{4 \sqrt{2}}\right) D_{12}
$$

We see that the coefficient of the linear term in $D_{12}$ cancels owing to contributions from $D_{\chi}^{2}$ and $\left|\Phi_{45}\right|^{2}$, and thus $V$ would increase for $D_{12} \neq 0$. This shows that the minimum of $V$ corresponding to case (a), with $D_{12}=D_{\psi}=0$, is preferred over that of case (b), with $D_{12}=-\sqrt{3} D_{\psi} \neq 0$. This in turn means that even for the more realistic, though complicated, case of solution I, there exist viable solutions for the pattern of VEVs for which only the family-universal contributions to squark masses,

\footnotetext{
$\ddagger$ The question of why the vacuum energy (cosmological constant) is so small or zero of course remains unanswered, as it is in all other analogous approaches leading to SUSY-breaking.
} 
arising through $D_{A}$ and $D_{\chi}$, survive, but the non-universal $D$-term contributions, associated with the family dependent $U(1)$ 's, vanish owing to minimization of the potential. While this result may not hold in general, it is remarkable that it does for the solutions considered here, which are viable. The conditions for emergence of this result, which are worth studying, will thus provide an important new guideline for selecting the string solutions, and the associated patterns of zeroth order VEVs which satisfy the $F$ and $D$-flat conditions.

The degeneracy in squark-masses, obtained as above at the string-unification scale, would of course be affected, as would be the ratios of the various gauge and Yukawa couplings, when they are extrapolated to low energies through the use of the renormalization group equations. This would not, however, have a significant effect at least on the degeneracy of the squarks of the first two families, which is relevant to the $K_{0}-\bar{K}_{0}$ transition 3 .

\section{sign of $\left\langle D_{A}\right\rangle$ - Contribution to Squares of Scalar Masses:}

Before discussing the origin of the mass-term $m$, one special property of both solutions I and II is worth noting. Given that the overall sign of $U(1)_{A}$ is chosen such that $\operatorname{Tr} Q_{A}$ is positive, the signs of the anomalous charges of all the fields are fixed. For instance, if the sign of $Q_{A}$ for squarks and/or sleptons in any solution happened to be negative, it must of course be discarded because the corresponding $D_{A}$ would lead to negative contributions to the (mass) ${ }^{2}$ of these fields (see Eq. (3.19) and thereby to a breaking of $S U(3)$-color and/or electric charge. As may be seen from tables 1 and 4, it is indeed remarkable that all the squarks and the sleptons have positive $Q_{A}$ for both solutions I and II.

One must still ensure that none of the other fields carrying color and/or electric

\footnotetext{
${ }^{\S}$ We should also add that even though $\left\langle D_{12}\right\rangle$ and $\left\langle D_{\psi}\right\rangle$ vanish at the level considered above, the VEVs of Higgs fields (like $H_{u}$ ) of electroweak scale will still induce a non-vanishing $\left\langle D_{12}\right\rangle \approx$ $Q_{12}\left(H_{u}\right)\left|\left\langle H_{u}\right\rangle\right|^{2}=(1 / \sqrt{2})\left|\left\langle H_{u}\right\rangle\right|^{2}$, and likewise a non-vanishing $\left\langle D_{\psi}\right\rangle$. This leads to a mass splitting $\left|\delta m_{\tilde{d}}^{2}-\delta m_{\tilde{s}}^{2}\right| \approx\left(g_{2}^{2} / 2\right)\left|\left(Q_{12}^{\tilde{d}}-Q_{12}^{\tilde{s}}\right)\right|\left\langle D_{12}\right\rangle \approx\left(g_{2}^{2} / 2\right)(1 /(2 \sqrt{2}))(1 / \sqrt{2})\left|\left\langle H_{u}\right\rangle\right|^{2} \approx(50 \mathrm{GeV})^{2} \leq$ $(1 / 186) \tilde{m}^{2}$, for $\tilde{m} \geq 700 \mathrm{GeV}$, where we have put $\left\langle H_{u}\right\rangle \sim 200 \mathrm{GeV}$. As discussed in the text, lack of squark degeneracy to this extent is of course compatible with the constraint of the real part of the $K_{0}-\bar{K}_{0}$ amplitude. We thank K.S. Babu for raising this point.
} 
charge acquire net negative (mass) ${ }^{2}$. Note first of all that the hidden sector fields $V_{i}$, $\bar{V}_{i}$ as well as $T_{i}$ and $\bar{T}_{i}$ (which are of course standard model singlets) have positive $Q_{A}$. The fields of possible concern for solution I are the conjugate pairs $\left(D_{45}, \bar{D}_{45}\right)$ and $\left(H_{21}, H_{22}\right)$ which carry color. Clearly one member of each such pair would have positive $Q_{A}$ but the other member would have negative $Q_{A}$, as do $D_{45}$ and $H_{21}$. Thus $\left\langle D_{A}\right\rangle$ would give negative (mass) ${ }^{2}$ to $D_{45}$ and $H_{21}$. We have, however, checked that higher dimensional operators for solution I as well as solution II give sufficient positive contribution to the (mass) ${ }^{2}$ of each member of these conjugate pairs, by utilizing the VEVs of standard model singlets of order $\sqrt{\xi}$ (as in Eq. (3.23)) and hidden sector condensates. This more than compensates for the negative contribution of $\left\langle D_{A}\right\rangle$. Specifically, for solution I, one obtains the operators $H_{21} H_{22} \xi_{1}$ and $D_{45} \bar{D}_{45} H_{19} H_{20} \xi_{1}^{3}$ at $N=3$ and $N=7$ respectively in the superpotential $W$. With $\xi_{1} \sim \sqrt{\xi}$ (see Eq. (3.23) $)$, and assuming that the $\left(H_{21} H_{20}\right)$ - pair condenses due to the $S U(5)_{H}$ force which confines at a scale of $10^{13}-10^{14} \mathrm{GeV}$ [30], these operators provide positive contributions to (mass) $)^{2}$ of $H_{21}$ and $H_{22}$ as well as of $D_{45}$ and $\bar{D}_{45}$, that far exceed the negative contributions of $\left\langle D_{A}\right\rangle$, which are of order $m^{2}$ [it is worth mentioning in advance that $m$ itself is induced only at $N=8$ by utilizing condensate of the same type as above (see next section)].

Now each member of a conjugate pair of Higgs doublets like $\left(h_{i}, \bar{h}_{i}\right)$ would also get negative contribution to its (mass) $)^{2} \sim(1 \mathrm{TeV})^{2}$ through $\left\langle D_{A}\right\rangle$. As mentioned in section 3 , the (mass) $)^{2}$ matrix of the Higgs-sector, including contributions from the string generated higher dimensional operators has many entries. This matrix has been analyzed in detail in Ref. [26], which showed that only one pair of doublets remains light, while the others acquire heavy or medium heavy masses. While a reanalysis of the Higgs mass-matrix including the $\left\langle D_{A}\right\rangle$-contributions of order $(1 \mathrm{TeV})^{2}$ deserves study in a separate work, it is clear that the latter contribution will affect only the light Higgs spectrum. [in general, it is possible that such a light Higgs may even acquire a VEV of order $1 \mathrm{TeV}$ due to the $\left\langle D_{A}\right\rangle$-contribution at a high scale; this by itself need not, however, be objectionable].

In summary, we note that the higher dimensional operators could not in any case 
have given masses to standard model non-singlet chiral fields like squarks and sleptons by using VEVs of only standard model singlet fields like those in Eq. (3.23) and hidden sector condensates. It is thus fortunate that these fields carry positive anomalous charges and thereby receive only positive contributions to their (mass) ${ }^{2}$ from $\left\langle D_{A}\right\rangle$ for both solutions I and II. On the other hand, higher dimensional operators can and do contribute positively to the (mass) ${ }^{2}$ of fields belonging to conjugate pairs, and more than compensate for negative contributions from $\left\langle D_{A}\right\rangle$ to such fields.

\section{Generating the mass term}

We now show how the mass term $m$ can arise through higher dimensional operators using hidden sector condensates. Consider first solution I (ref.[13]). The allowed operators up to $N=8$ are listed below:

at order $\mathrm{N}=5$,

$$
\begin{array}{ll}
V_{2} \bar{V}_{2} \Phi_{45} \Phi_{2}^{-} \xi_{1}, & V_{1} \bar{V}_{1} \Phi_{45} \bar{\Phi}_{1}^{+} \xi_{2}, \\
T_{2} \bar{T}_{2} \Phi_{45} \Phi_{2}^{+} \xi_{1}, & T_{1} \bar{T}_{1} \Phi_{45} \bar{\Phi}_{1}^{-} \xi_{2}
\end{array}
$$

at order $\mathrm{N}=7$,

$$
\begin{gathered}
T_{2} \bar{T}_{3} V_{3} \bar{V}_{2} \Phi_{45} \Phi_{45} \bar{\Phi}_{13}, \\
T_{1} \bar{T}_{2} V_{1} \bar{V}_{2} \Phi_{45} \Phi_{45} \xi_{1}, \\
T_{2} \bar{T}_{1} V_{1} \bar{V}_{2} \Phi_{45} \Phi_{45} \xi_{2} \\
\left.V_{2} \bar{V}_{2} \Phi_{45} \Phi_{2}^{-} \xi_{1}\left[\left(\frac{\partial W_{3}}{\partial \xi_{3}}\right)+\xi_{i} \xi_{i}+\Phi_{13} \bar{\Phi}_{13}+\Phi_{23} \bar{\Phi}_{23}\right)\right], \\
\left.V_{1} \bar{V}_{1} \Phi_{45} \bar{\Phi}_{1}^{+} \xi_{2}\left[\left(\frac{\partial W_{3}}{\partial \xi_{3}}\right)+\xi_{i} \xi_{i}+\Phi_{13} \bar{\Phi}_{13}+\Phi_{23} \bar{\Phi}_{23}\right)\right], \\
V_{2} \bar{V}_{2} \Phi_{45} \bar{\Phi}_{2}^{+} \xi_{1}\left(\frac{\partial W_{3}}{\partial \Phi_{12}}\right), \\
V_{1} \bar{V}_{1} \Phi_{45} \Phi_{1}^{-} \xi_{2}\left(\frac{\partial W_{3}}{\partial \bar{\Phi}_{12}}\right)
\end{gathered}
$$

and at order $\mathrm{N}=8$,

$$
\bar{T}_{2} T_{3} V_{3} \bar{V}_{2} \Phi_{45} \Phi_{45} \bar{\Phi}_{13} \xi_{1}
$$




$$
\begin{gathered}
T_{1} \bar{T}_{3} V_{1} \bar{V}_{3} \Phi_{45} \Phi_{45} \bar{\Phi}_{23} \xi_{2}, \\
T_{2} \bar{T}_{3} V_{2} \bar{V}_{3} \Phi_{45} \Phi_{45} \bar{\Phi}_{13} \xi_{1}, \\
T_{3} \bar{T}_{1} V_{3} \bar{V}_{1} \Phi_{45} \Phi_{45} \bar{\Phi}_{23} \xi_{2} . \\
\Phi_{45} \bar{\Phi}_{45} T_{3} \bar{T}_{3} \Phi_{45} \bar{\Phi}_{13} \bar{\Phi}_{3}^{-} \xi_{1}, \\
\Phi_{45} \bar{\Phi}_{45} T_{3} \bar{T}_{3} \Phi_{45} \bar{\Phi}_{13} \bar{\Phi}_{3}^{-} \xi_{2},
\end{gathered}
$$

We see from (4.1), that given the pattern of VEVs listed in eq. (3.23), no mass term can arise at $N=5$. At $N=7$ bilinear mass-terms like $\Phi_{45}^{2}$ could arise, only if non-diagonal hidden sector condensates like $\left\langle T_{2} \bar{T}_{3}\right\rangle$ as well as $\left\langle V_{3} \bar{V}_{2}\right\rangle$ could form. For ordinary QCD, such condensates (like $\langle\bar{d} s\rangle$ ) are forbidden. Even if they do form, the magnitude of such mass-terms is of order $\left(\Lambda_{5} / M_{\mathrm{st}}\right)^{2}\left(\Lambda_{3} / M_{\mathrm{st}}\right)^{2} M^{\prime}$, where $\Lambda_{5}$ and $\Lambda_{3}$ represent the $S U(5)_{H}$ and $S U(3)_{H}$ confinement scales, respectively, and $M^{\prime}$ is of order $\sqrt{\xi}$, representing the scale of the VEVs of the singlet $\Phi$-fields in eq. (3.23). Taking typical values of $\Lambda_{5} \sim 10^{13}-10^{14} \mathrm{GeV}$ and $\Lambda_{3} \sim 10^{8}-10^{10} \mathrm{GeV}$, which are suggested by renormalization group analysis for solution I [30]. With $M_{\mathrm{st}} \sim 10^{18} \mathrm{GeV}$ and $M^{\prime} \sim(1 / 3-1) \times 10^{17} \mathrm{GeV}$, such mass-terms are $\leq 10^{-9} \mathrm{GeV}$, and are thus insignificant for phenomenological purposes. Assuming that at least the diagonal condensates in the hidden sector, like $\left\langle T_{3} \bar{T}_{3}\right\rangle$ form, the only relevant mass-term is given by the $N=8$ term shown in Eq. (4.5). This yields a mass term $m \Phi_{45} \bar{\Phi}_{45}$ which is neutral with respect to all charges and is of order :

$$
m\left(\Phi_{45}\right)=m\left(\bar{\Phi}_{45}\right) \sim\left(\frac{\Lambda_{5}}{M_{s t}}\right)^{2}\left(\frac{M^{\prime}}{M_{s t}}\right)^{3} M^{\prime}
$$

It is remarkable that $m$ receives contributions only at $N \geq 8$. Since $\Lambda_{5}$ is 4 to 5 orders of magnitude smaller, and $M^{\prime}$ is about 10 to 30 times smaller than $M_{s t}$, it is clear that the SUSY mass splitting $m$ is naturally strongly suppressed compared to $M_{s t}$. As regard its numerical value, for values of $\Lambda_{5}$ and $M^{\prime}$ lying in the range mentioned above, i.e. $\left(\Lambda_{5} / M_{s t}\right)^{2} \sim 10^{-8}-10^{-10},\left(M^{\prime} / M_{s t}\right)^{3} \sim 10^{-4}$ and $M^{\prime} \sim(1 / 2)\left(10^{17} \mathrm{GeV}\right)$, say - which are most plausible, we get :

$$
m \sim\left(\frac{1}{2}-50\right) \mathrm{TeV} .
$$


Since $m$ represents the scale of supersymmetry breaking and thus the mass scale of the Higgs scalars, and in turn of $m_{W}$, we see that the string solutions under consideration do explain why the electroweak scale is so much smaller than the string scale.

\section{Some phenomenological aspects}

An interesting phenomenological distinction, first between the two solutions I and II, considered above, is worth noting. Although the anomalous charge $Q_{A}$ for solution I [13], given by eq. (2.2), is family - universal, it distinguishes between different members of a family (see table 1), and therefore leads to intra-family mass splittings among the scalars. Including contributions from the leading $D_{A}$ term only, these are represented, by the following relative values, at the scale of $\sqrt{\xi}$, for any given family :

$$
\left[m^{2}\left(\tilde{Q}_{L}\right): m^{2}\left(\tilde{u}_{R}\right): m^{2}\left(\tilde{d}_{R}\right): m^{2}(\tilde{L}): m^{2}\left(\tilde{e}_{R}\right)\right]_{D_{A}}=3: 1: 3: 1: 1 \quad \text { soln I }
$$

For solution II [14], on the other hand, $Q_{A}$ given by eq. (2.6), is the same for all members of a family. As a result, in so far as the leading contributions from the $D_{A}$-term, one obtains intra-family universal scalar masses at the scale $\sqrt{\xi}$, which are given by :

$$
\left[m^{2}\left(\tilde{Q}_{L}\right): m^{2}\left(\tilde{u}_{R}\right): m^{2}\left(\tilde{d}_{R}\right): m^{2}(\tilde{L}): m^{2}\left(\tilde{e}_{R}\right)\right]_{D_{A}}=1: 1: 1: 1: 1 \text { soln II }
$$

Thus, eventually empirical study of the squark spectrum can in fact distinguish between the two string solutions I and II.

It is also worth noting that both string solutions I and II lead to approximately universal scalar masses (at the scale of $\sqrt{\xi}$ ) for all three families. At the same time, owing to spontaneously induced asymmetric Higgs mass spectrum (see discussion in section 3) they lead to hierarchical fermion masses [26]. By contrast, the model of ref. [5] assumes that $U(1)_{A}$ couples universally only to the first two families and thus predicts heavier squark masses $(\sim 5 \mathrm{TeV})$ for the first two families and lighter mass $(\sim 500 \mathrm{GeV})$ for the stop, while the gauginos are lighter still $(\sim 50-100 \mathrm{GeV})$. It has been pointed out in ref. [31], however that models of this class [5, 7] with 
two-family universality (of the anomalous $U(1)$ ) typically lead to color and electric charge-breaking, assuming that the spectrum of the type noted above is generated near the Planck or the GUT-scale. This is because contributions from two-loop renormalization group evolution to the scalar masses contain terms which are proportional to the larger squark (mass) ${ }^{2}$ of the first two families, and are negative. This negative contribution turns the initially smaller positive stop $(\text { mass })^{2} \sim(500 \mathrm{GeV})^{2}$ to negative values at the $\mathrm{TeV}$-scale and thereby induces color and charge-breaking. Models with three-family universality (of the anomalous $U(1)$ ), as discussed here, do not however face this problem because the squarks of all three families are nearly degenerate, with only moderately heavy masses. Several considerations suggest that they should have a mass of about $1 \mathrm{TeV}$, within a factor of two, either way, at the electroweak scale. This reduces the RGE-induced negative contribution to the squark $(\text { mass })^{2}$ by about an order of magnitude, while increasing the initial positive value of $m_{\tilde{t}}^{2}$ at the GUT or the string scale, compared to the case of two-family universality. It thereby eliminates the problem of color and charge-breaking. Thus it seems that phenomenological considerations favor three-family universality, for SUSY-breaking through anomalous $U(1)$. It is intriguing that string-solutions of the type considered here yield precisely that.

\section{Remarks on Supersymmetry Breaking Through Anoma- lous $U(1)$}

Before concluding the following remarks are in order.

(1) Desirability and Origin of Family-Permutation Symmetry: If supersymmetry breaking occurs entirely or dominantly through an anomalous $U(1)$, as noted in the last section, the need to avoid color and charge-breaking suggests that the $U(1)_{A}$ must be universal with respect to all three families. At the same time, the hierarchical masses and mixings of the three families suggest that there ought to exist flavor or horizontal gauge symmetries, beyond GUTs in the underlying theory, which distinguish between the families and are ultimately responsible for the hierarchy in 
their masses. The virtues of flavor symmetries in the string context, (like $U_{1}$ to $U_{6}$ for solution I and $U_{1}$ to $U_{5}$ for solution II) in this regard has been noted in previous works [34, 26], and in the non-string context by several authors [35]. Furthermore, these flavor symmetries have been shown to play a crucial role in addressing certain naturalness problems of supersymmetry, such as the enormous suppressions of (a) the $d=4$ and $d=5$ rapid proton-decay operators [17, 18], (b) $\nu_{L}-\tilde{\mathrm{H}}$ mixing mass [19], and (c) the mass-term $m$ of relevant fields which triggers SUSY-breaking(see section 4). We suspect that they are also responsible for the desired suppression of the $\mu$-parameter.

As alluded to above, such family-dependent flavor symmetries, which are clearly absent in GUTs, do in fact emerge quite generically in string theory (e.g. from an underlying $S O(44)$ in the free fermionic construction). Now, typically, at least a subset of these family-dependent $U(1)^{\prime} s$ would appear to be anomalous in a general basis (compare with $U_{1}$ to $U_{6}$ for solution I and $U_{1}$ to $U_{3}$ for solution II); these can be grouped to give anomaly-free combinations (like $U_{\psi}, U_{12}, U_{\zeta}, U_{45}$ and $U_{\chi}$ in solution I (see eqs. (2.3 2.5)); except for one unique combination that remains anomalous and gives the $U(1)_{A}$ (see eq. (2.2)). Similar situations arise in all other semi-realistic string-derived models which exist to date; see e.g. Refs. 19, 10, 11, 12, 13, 14, 15, 32, 33].

The question then arises how can there be these flavor-symmetries, which distinguish between the families and are thus family - non-universal, and yet there be an anomalous $U(1)_{A}$, arising from linear combinations of the same flavor-symmetries, which is family universal ? The only way, it appears to us, is that the flavor symmetries, although family-dependent, must still respect the permutation symmetry (mentioned in secs 1 and 2) with respect to all three families. In this case $U(1)_{A}$ would automatically be family universal, as borne out by the examples of eqs. (2.2) and (2.7).

Thus, SUSY-breaking through $U(1)_{A}$, together with the presence of flavor symmetries, seem to suggest the need for the stated permutation symmetry. As stated in section 2, such a symmetry is in fact an internal property of at least the NAHE set 
of boundary condition basis vectors $\left\{\mathbf{1}, S, b_{1}, b_{2}, b_{3}\right\}$, for which the cyclic permutation symmetry corresponds simply to the symmetry between the three twisted sectors of the $Z_{2} \times Z_{2}$ orbifold, which arise from the sectors $b_{1}, b_{2}$ and $b_{3}$, respectively. In view of the importance of the permutation symmetry, as noted above, it would be interesting to know whether such a symmetry could arise without utilizing the NAHE set. While it is premature to ascertain the answer to this question at present, we note that there do in fact exist three-generation string solutions based on the free fermionic construction which utilize only a subset $\left\{\mathbf{1}, S, b_{1}, b_{2}\right\}$ of the NAHE set of basis vectors (see e.g. Ref. [15]); these, however, do not possess the cyclic permutation symmetry.

It is also worth noting that while the NAHE set (by itself) yields the permutation symmetry, it of course does not guarantee that the symmetry will be retained in the presence of additional boundary condition basis vectors, which are needed to reduce the number of generations from 48 to 3 . As noted before, all four solutions exhibited respectively in Refs. [9], [11], [13] and [14], utilize the NAHE set; but only the last two retain the permutation symmetry, while the first two do not. Thus, string solutions of the type obtained in Refs. [13] and [14 appear to be particularly suited to break supersymmetry through an anomalous $U(1)$, while providing the squark degeneracy.

(2) A Scenario of Combined Anomalous $U(1)$-Dilaton SUSY breaking: It has been noted in section 3 (see discussion following eq. (3.22)) that if SUSYbreaking proceeds entirely through a family-universal $U(1)_{A}$, it would lead to the desired squark degeneracy, but it is likely to lead to unacceptably light gluinos. At this point, two apparently unrelated issues, both associated with the dilaton, are worth recalling. First, there is the well known problem of dilaton-stabilization. Regardless of whether SUSY-breaking utilizes the VEV of the dilaton-auxiliary component, $F_{S}$, or not, one needs to avoid its generic weak-coupling runaway behavior (i.e. $S \rightarrow \infty$ ), and obtain instead a stable minimum of its potential at a value of $S=S_{0} \sim 10-20$, rather than at infinity or 1 [for a discussion of this issue and references to various attempts for its resolution in the field-theory and string-theory/M-theory context, see e.g. Ref. [36] and references therein]. Second, if SUSY-breaking is dominated by the VEV of $F_{S}$, it seems that one would encounter the problem of color and electric 
charge - breakings (see e.g. last paper of Ref. [2]).

It seems to us, however, that in a mutually coupled system such as ours, supersymmetry-breaking may well proceed through multiple sources whose effects on soft masses may in general be comparable. In some cases, one of the sources may be viewed as primary and the other(s) secondary, and the former may in fact induce the latter. We have in mind the mutual couplings between (a) the dilaton superfield $S$ and the non-perturbatively generated hidden sector $S U(5)_{H}$ gaugino and matter condensates (whose scale $\Lambda_{5}$ is proportional to $\mathrm{e}^{\left(-S / 2 b_{5}\right)}$ ) on the one hand, and (b) that between $S$ and the anomalous $U(1)_{A}$ gauge field via the Green-Schwarz term that generates the Fayet-Iliopoulos term $\xi$ on the other hand (see e.g. Ref. [6]). Because of the mutual couplings, these three components - i.e. the dilaton, the hidden sector condensates and the anomalous $U(1)$ - can influence each other's role significantly and thereby the nature of SUSY-breaking. The task at hand therefore is the minimization of the full effective potential for this coupled system, which receives contributions from (a) and (b), as well as possibly from additional non-perturbative terms in the Kähler potential. Such a minimization is to be carried out in the presence of the SUSY-preserving VEVs of standard model singlet fields $\left\{\Phi^{i}\right\}$ (see e.g. (3.23), which are induced because of the Fayet-Iliopoulos term, and which generate the mass term $m$, by utilizing the hidden sector condensates, as in section 4 .

One particularly attractive possibility which we defer for further study is this. The hidden sector condensates, involving in general matter and gaugino pairs, in conjunction with the SUSY-preserving VEVs of the $\left\{\Phi_{i}\right\}$-fields generate the mass term $m$ as in section 4, which in turn triggers SUSY-breaking through a family universal $U(1)_{A}$, as in section 3 . This could provide at least a major contribution to squark-masses $\sim(1 / 3-2) \mathrm{TeV}$ (say), which is approximately family universal. Simultaneously, the coupling of the dilaton to the hidden sector condensates as well as to the gauge field of $U(1)_{A}$, together perhaps with non-perturbative terms in the Kähler potential, stabilizes the dilaton at a desired value $S_{0}$, while inducing a VEV for the dilaton auxiliary field $\left\langle F_{S_{0}}\right\rangle \neq 0$.

Such a scenario, if it can be realized, would have the following advantages: 
(i) The dilaton-induced SUSY breaking $\left(\left\langle F_{S}\right\rangle \neq 0\right)$ would not upset the squarkdegeneracy that was obtained through the family-universal $U(1)_{A}$, because the dilaton contributes universally to the scalar masses (barring smaller loop-corrections). (ii) Since dilaton SUSY-breaking assigns comparable masses to squarks and gauginos (unlike $\left.U(1)_{A}\right)$, following the relations $\left(\Delta m_{\tilde{g}}\right)_{F_{S}}=\sqrt{3}\left(\delta m_{\tilde{q}}\right)_{F_{S}}=\sqrt{3}\left(m_{3 / 2}\right)_{F_{S}}$, however, it could provide the leading contribution to gluino and wino masses: $\left(\Delta m_{\tilde{g}}\right)_{F_{S}} \approx \sqrt{3}(1$ to few $)(100) \mathrm{GeV}$ (say) - while providing significant contributions to squark-masses $\circledast$. This would remove the problem of light gluino for $U(1)_{A}-\mathrm{SUSY}$ breaking. (iii) SUSY breaking through the combination of a universal $U(1)_{A}$ and dilaton mechanisms, as described above, would of course avoid the danger of color and charge-breaking, that confronts the scenario of purely dilaton dominated SUSY breaking (see the last paper in Ref. [2]). In short, the combined SUSY-breaking mechanism involving a family-universal $U(1)_{A}$ and the dilaton has the advantage that each component cures the vices of the other, without upsetting any of its virtues. The feasibility of this combined source of SUSY-breaking, including its effects on dilaton - stabilization, is clearly worth further study [37.

(3) Unlike the models of Refs. [5] and [7], which introduce very few fields and just the single anomalous $U(1)_{A}$, but without any accompanying flavor-symmetries, string solutions generically contain many fields (see e.g. tables 1-3 and 4-6) and typically several $U(1)$ 's, some of which are family dependent. In spite of this more elaborate (though fixed) structures, it is interesting that minimization for the string solutions considered here, led to a hierarchical pattern of soft masses (see Eq. (3.22)) which is very similar to the cases of Refs. [5]-[7], barring of course the distinction of three versus two-family degeneracy that arises from the differences in $U(1)_{A}$ (see section 5). In particular, it is remarkable that, for the string solutions considered here, the VEVs of $D_{\alpha}$ 's associated with family-dependent $U(1)_{\alpha}$ 's turned out to vanish, owing to the requirement of a relatively global minimum of the potential. But for

${ }^{*}$ While $\left\langle F_{S}\right\rangle$ and $\left\langle D_{A}\right\rangle$-contributions to squark-masses may be comparable, their relative proportion will be constrained by the need to avoid color and charge-breaking (see Ref. [2]). Quite clearly, dominant contribution from $\left\langle F_{S}\right\rangle$ would be excluded on this ground. 
this feature, SUSY-breaking through anomalous $U(1)$ in string models would not be viable.

(4) The Necessary Ingredients: From the preceding discussion and those in sections 3 and 4 , it is clear that the following set of ingredients are in fact needed in order that supersymmetry breaking through anomalous $U(1)$ can be implemented consistently, especially in the string-context: (i) family-universality of the $U(1)_{A}$ and therefore the family permutation symmetry of the flavor gauge symmetries as discussed above; (ii) suitably suppressed effective mass term $m$ of relevant fields carrying the anomalous charge; (iii) positivity of the anomalous charges of the chiral squark and slepton fields; and (iv) vanishing (or adequate suppression) of the undesirable $D$-terms, associated with family-dependent $U(1)$ 's, because of minimization of the potential. It seems truly remarkable that there do exist string solutions, as discussed here, for which all four ingredients are realized. If the anomalous $U(1)$ would turn out to provide an important source of SUSY-breaking, realization of these necessary features, as well as meeting the non-trivial constraints from issues such as proton longevity [17, 18 and fermion masses and mixings, together would clearly provide a very useful set of criteria in severely limiting the desired class of solutions from the vast set that is available.

(5) Gravitino Mass: An important effective parameter of SUSY-breaking is the mass of the gravitino. With SUSY-breaking through only anomalous $U(1)$, as described in section 3 , the gravitino would receive a mass $m_{3 / 2} \sim\left\langle F\left(\bar{\Phi}_{45}\right)\right\rangle / M_{P l} \approx$ $m \sqrt{\epsilon} \approx\left(m_{\tilde{q}} / 10\right) \sim(1$ to few $)(100 \mathrm{GeV})$. With additional sources of SUSY-breaking, involving for example the dilaton and possibly hidden sector condensates, as motivated above, $m_{3 / 2}$ would get further contributions. While the relative contributions of these different sources of SUSY-breaking to $m_{3 / 2}$, squark and gluino masses are not easy to ascertain at present, we would still expect $m_{3 / 2}$ to lie in the $100 \mathrm{GeV}$ to a few TeV-range. 


\section{Summary}

In summary, an anomalous $U(1)$ gauge symmetry, together with an effective massterm for certain relevant fields, offers a very simple mechanism to implement SUSYbreaking. It is shown here that this mechanism can in fact be derived consistently, leaving aside the question of dilaton-stabilization, from an underlying string theory. While string solutions invariably possess an anomalous $U(1)$ symmetry, the require-

ment of three family universality of squark masses and therefore of $U(1)_{A}$ is not easy to satisfy. We have shown that there do exist certain three generation string solutions for which supersymmetry breaking through an anomalous $U(1)$ leads to both the desired three-family squark-degeneracy and the large hierarchy between the string and the electroweak scales. More specifically we have noted that these solutions, in contrast to most, possess a cyclic permutation symmetry between the three families, which automatically yields a set of non-anomalous but family-dependent flavor gauge symmetries on the one hand, and a family-universal anomalous $U(1)$ gauge symmetry on the other hand. It is the non-anomalous flavor symmetries, unavailable in GUTs, which are ultimately responsible for hierarchical fermion masses and CKM mixings as well as for the desired suppression of both the rapid proton decay operators and of the effective SUSY-breaking mass parameter $m$. The anomalous $U(1)_{A}$ is, however, family universal. In other words it is not a horizontal symmetry, unlike the models of Refs. [5] and [7], and it is this feature that makes it suitable for the purposes of SUSY-breaking without encountering color and electric charge breaking. We further note that family universality of the anomalous $U(1)$ has also been found to be desirable in recent attempts to fit the fermion mass spectrum by the use of Abelian horizontal symmetries [38].

We have remarked that the family permutation symmetry of the solutions of interest [13, 14], is a joint consequence of (a) the NAHE set of boundary condition basis vectors which corresponds to a $Z_{2} \times Z_{2}$ orbifold compactification, and (b) the special choice of additional boundary condition basis vectors, beyond the NAHE set, which serve to reduce the number of generations from 48 to 3 . While suitable 
variations in (b) could still allow the permutation symmetry to be retained, it is far from clear whether the same can still be realized without the NAHE set.

As regards the issue of supersymmetry-breaking, we have noted that the marriage of the two sources for such a breaking - i.e. through $U(1)_{A}$ and through the dilaton - if it can be realized, would be most attractive because it would combine the advantages of both, while each would remove the disadvantage of the other. Realization of this combined mechanism, would thus be of major importance.

To conclude, if the $D$-term of the anomalous $U(1)$ makes a major contribution to squark masses, it must be family-universal. In this case, if the NAHE set turns out to be a necessary ingredient to obtain a family universal anomalous $U(1)$, it would be an indication that the string vacuum is in the vicinity of the $Z_{2} \times Z_{2}$ orbifold, with the standard embedding of the gauge connection. Thus, the question about the necessity of the NAHE set for obtaining the family-permutation symmetry is an interesting and important one, worth further study.

It is a pleasure to thank I. Antoniadis, G. Cleaver, C. Kolda, J. March-Russell, A. Pomarol, P. Ramond and especially K.S. Babu, G. Dvali and H. Murayama for useful discussions. We also wish to thank the hospitality of the CERN theory division, where part of this work was done. This work was supported in part by DOE Grant No. DE-FG-0586ER40272 (AEF) and in part by PHY-9119745 (JCP). The research of JCP was supported in part by a Distinguished Faculty Research Fellowship awarded by the University of Maryland.

\section{Note added in proof:}

After the submission of our paper, an interesting work has recently appeared [39], where the authors demonstrate the emergence of the combined anomalous $U(1)-$ dilaton SUSY-breaking picture (i.e. $\left\langle D_{A}\right\rangle \neq 0$ and $\left\langle F_{S}\right\rangle \neq 0$ ), which was motivated in sec. 6 of our paper, because of the mutual coupling between the two systems and also on phenomenological grounds. While the dilaton-contribution seems to dominate over that of $\left\langle D_{A}\right\rangle$ in the cases studied in [39], as the authors note, the relative contributions of $\left\langle D_{A}\right\rangle$ and $\left\langle F_{S}\right\rangle$ to squark masses would of course depend upon the manner of dilaton-stabilization. Following remarks in sec. 6, a desirable 
solution would seem to be one in which the $\left\langle D_{A}\right\rangle$ contribution is at least comparable to that of $\left\langle F_{S}\right\rangle$, so that the problem of color-charge breaking is avoided. This issue needs further study.

\section{References}

[1] M. Dine, R. Rohm, N. Seiberg and E. Witten, Phys. Lett. B156 (1985) 55;

J.P. Derendinger, L.E. Ibanez and H.P. Nilles, Phys. Lett. B155 (1985) 65;

H.P. Nilles, Phys. Lett. B115 (1982) 193;

For the dilaton runaway problem in this scenario, see M. Dine and N. Seiberg, Phys. Lett. B162 (1985) 229.

[2] V. Kaplunovsky and J. Louis, Phys. Lett. B306 (1993) 269;

R. Barbieri, J. Louis and M. Moretti, Phys. Lett. B312 (1993) 451;

For a recent review of phenomenological aspects of dilaton-dominated SUSYbreaking, see e.g.: A. Brignole, L.E. Ibanez and C. Munoz, hep-ph/9707209, and references therein;

For discussion of color and charge-breaking, that arises in this case due to unboundness of the potential from below, see J. Casas, A. Llyeda and C. Munoz, Phys. Lett. B380 (1996) 59.

[3] M. Dine, A.E. Nelson and Y. Shirman, Phys. Rev. D51 (1995) 1362;

M. Dine, A.E. Nelson, Y. Nir and Y. Shirman, Phys. Rev. D53 (1996) 2658;

M. Dine, Y. Nir and Y. Shirman, Phys. Rev. D55 (1997) 1501.

[4] Aspects of these ideas were initiated in:

I. Antoniadis, John Ellis, A.B. Lahanas and D.V. Nanopoulos, Phys. Lett. B241 (1990) 24;

A.E. Faraggi, E. Halyo, Int. J. Mod. Phys. A11 (1996) 2357;

Relevance of suitable mass-terms in inducing SUSY-breaking was pointed out by, P. Fayet, Nucl. Phys. B90 (1975) 104.

[5] G. Dvali and A. Pomarol, Phys. Rev. Lett. 77 (1996) 3728. 
[6] P. Binetruy and E. Dudas, Phys. Lett. B389 (1996) 503.

[7] R.N. Mohapatra and A. Riotto, Phys. Rev. D55 (1997) 4262.

[8] A.E. Faraggi, Phys. Lett. B387 (1997) 775.

[9] I. Antoniadis, J. Ellis, J. Hagelin and D.V. Nanopoulos, Phys. Lett. B231 (1989) 65.

[10] A.E. Faraggi, D.V. Nanopoulos, and K. Yuan, Nucl. Phys. B335 (1990) 347.

[11] I. Antoniadis, G.K. Leontaris and J. Rizos, Phys. Lett. B245 (1990) 161;

G.K. Leontaris, Phys. Lett. B372 (1996) 212.

[12] I. Antoniadis, J. Ellis, S. Kelley and D.V. Nanopoulos, Phys. Lett. B272 (1991) 31 ;

J.L. Lopez, D.V. Nanopoulos and K. Yuan, Nucl. Phys. B399 (1993) 654.

[13] A.E. Faraggi, Phys. Lett. B278 (1992) 131.

[14] A.E. Faraggi, Phys. Lett. B274 (1992) 47.

[15] S. Chaudhoury, G. Hockney and J. Lykken, Nucl. Phys. B469 (1996) 357.

[16] H. Kawai, D.C. Lewellen, and S.-H.H. Tye, Nucl. Phys. B288 (1987) 1;

I. Antoniadis, C. Bachas, and C. Kounnas, Nucl. Phys. B289 (1987) 87.

[17] A.E. Faraggi, Nucl. Phys. B428 (1994) 111; Phys. Lett. B339 (1994) 223;

J. Ellis, A.E. Faraggi and D.V. Nanopoulos, hep-th/9709049.

[18] J.C. Pati, Phys. Lett. B388 (1996) 532; Proceedings of the International Workshop on Future Prospects of Baryon Instability, Oak Ridge TN, March 1996, hep-ph/9611371.

[19] A.E. Faraggi and J.C. Pati, Phys. Lett. B400 (1997) 314. 
[20] P. Ginsparg, Phys. Rev. D197 (1987) 139;

V. Kaplunovsky, Nucl. Phys. B307 (1988) 145;

I. Antoniadis, J. Ellis, R. Lacaze and D.V. Nanopoulos, Phys. Lett. B268 (1991) 188 ;

K.R. Dienes and A.E. Faraggi, Nucl. Phys. B457 (1995) 409.

[21] L. Dixon, E. Martinec, D. Friedan and S. Shenker, Nucl. Phys. B282 (1987) 13;

M. Cvetic, Phys. Rev. Lett. 59 (1987) 2829.

[22] S. Kalara, J.L. Lopez and D.V. Nanopoulos, Nucl. Phys. B353 (1991) 650.

[23] A.E. Faraggi and D.V. Nanopoulos, Phys. Rev. D48 (1993) 3288;

A.E. Faraggi, Nucl. Phys. B387 (1992) 239; hep-th/9708112;

G.B. Cleaver and A.E. Faraggi, hep-ph/9711339.

[24] A.E. Faraggi, Phys. Lett. B326 (1994) 62.

[25] A.E. Faraggi and M. Masip, Phys. Lett. B388 (1996) 524.

[26] A.E. Faraggi, Nucl. Phys. B403 (1993) 101; Nucl. Phys. B407 (1993) 57;

A.E. Faraggi and E. Halyo, Nucl. Phys. B416 (1994) 63.

[27] A.E. Faraggi, Nucl. Phys. B487 (1997) 55.

[28] M. Dine, N. Seiberg and E. Witten, Nucl. Phys. B289 (1987) 585;

J.J. Atick, L.J. Dixon and A. Sen, Nucl. Phys. B292 (1987) 109;

S. Cecotti, S. Ferrara and M. Villasante, Int. J. Mod. Phys. A2 (1987) 1839;

M. Dine, I. Ichinose and N. Seiberg, Nucl. Phys. B293 (1988) 253.

[29] See e.g. M. Dine, A. Kagan and S. Samuel, Phys. Lett. B243 (1990) 250;

F. Gabbiani, E. Gabrielli, A. Masiero and L. Silvestrini, Nucl. Phys. B477 (1996)

321 , and references therein;

For a recent improved calculation, including QCD corrections, see J. Bagger, K.

Matchev and R. Zhang, hep-ph/9707223.

[30] A.E. Faraggi and E. Halyo, Phys. Lett. B307 (1993) 311. 
[31] N. Arkani-Hamed and H. Murayama, Phys. Rev. D56 (1997) 6733.

[32] L.E. Ibanez, J.E. Kim, H.P. Nilles and F. Quevedo, Phys. Lett. B191 (1987) 282 ;

D. Bailin, A. Love and S. Thomas, Phys. Lett. B194 (1987) 385;

A. Font, L.E. Ibanez, F. Quevedo and A. Sierra, Nucl. Phys. B331 (1990) 421.

[33] A.A. Maslikov, S.M. Sergeev and G.G. Volkov, Phys. Rev. D50 (1994) 7440;

D. Finnell, Phys. Rev. D53 (1996) 5781;

Z. Kakushadze and S.H.H. Tye, Phys. Rev. Lett. 77 (1996) 2612; Phys. Rev. D55 (1997) 7896;

Z. Kakushadze, G. Shiu, S.H.H. Tye and Yan Vtorov-Karevsky, hep-ph/9710149.

H.D. Dahmen, A.A. Maslikov, I.A. Naumov, T. Stroh and G.G. Volkov, hepth/9711192.

[34] J.L. Lopez and D.V. Nanopoulos, Nucl. Phys. B338 (1990) 73; Phys. Lett. B251 (1990) 73 ;

I. Antoniadis, J. Rizos and K. Tamvakis, Phys. Lett. B278 (1992) 257;

B.C. Allanach, S.F. King, G.K. Leontaris, S. Lola, Phys. Rev. D56 (1997) 2632;

J. Ellis, G.K. Leontaris, S. Lola and D.V. Nanopoulos, hep-ph/9711476.

[35] For an incomplete set of references, see e.g.

P. Pouliot and N. Seiberg, Phys. Lett. B318 (1993) 169;

D. Kaplan and M. Schmaltz, Phys. Rev. D49 (1994) 3741;

L. Hall and H. Murayama, Phys. Rev. Lett. 75 (1995) 3985;

E. Dudas, S. Pokorski, C.A. Savoy, Phys. Lett. B356 (1995) 45;

R. Barbieri, G. Dvali and L.J. Hall, Phys. Lett. B377 (1996) 76

P. Binetruy, S. Lavignac, P. Ramond, Nucl. Phys. B477 (1996) 353;

P. Binetruy, N. Irges, S. Lavignac, P. Ramond, Phys. Lett. B403 (1997) 38.

[36] See e.g. V. Kaplunovsky and J. Louis hep-th/9708049, and references therein.

[37] For a recent attempt in this direction, made in the context of a non-string model, see Z. Lalak, hep-th/9708410. 
[38] J.K. Elwood, N. Irges and P. Ramond, hep-ph/9705270.

[39] N. Arkani-Hamed, M. Dine and S.P. Martin, hep-ph/9803432. 


\begin{tabular}{|c|c|c|c|c|c|c|c|c|c|c|c|c|c|}
\hline$F$ & SEC & $S U(3) \times S U(2)$ & $Q_{C}$ & $Q_{L}$ & $Q_{1}$ & $Q_{2}$ & $Q_{3}$ & $Q_{4}$ & $Q_{5}$ & $Q_{6}$ & $S U(5) \times S U(3)$ & $Q_{7}$ & $Q_{8}$ \\
\hline$L_{1}$ & \multirow[t]{6}{*}{$b_{1}$} & $(1,2)$ & $-\frac{3}{2}$ & 0 & $\frac{1}{2}$ & 0 & 0 & $\frac{1}{2}$ & 0 & 0 & $(1,1)$ & 0 & 0 \\
\hline$Q_{1}$ & & $(3,2)$ & $\frac{1}{2}$ & 0 & $\frac{1}{2}$ & 0 & 0 & $-\frac{1}{2}$ & 0 & 0 & $(1,1)$ & 0 & 0 \\
\hline$d_{1}$ & & $(\overline{3}, 1)$ & $-\frac{1}{2}$ & -1 & $\frac{1}{2}$ & 0 & 0 & $-\frac{1}{2}$ & 0 & 0 & $(1,1)$ & 0 & 0 \\
\hline$N_{1}$ & & $(1,1)$ & $\frac{3}{2}$ & -1 & $\frac{1}{2}$ & 0 & 0 & $-\frac{1}{2}$ & 0 & 0 & $(1,1)$ & 0 & 0 \\
\hline$u_{1}$ & & $(\overline{3}, 1)$ & $-\frac{1}{2}$ & 1 & $\frac{1}{2}$ & 0 & 0 & $\frac{1}{2}$ & 0 & 0 & $(1,1)$ & 0 & 0 \\
\hline$e_{1}$ & & $(1,1)$ & $\frac{3}{2}$ & 1 & $\frac{1}{2}$ & 0 & 0 & $\frac{1}{2}$ & 0 & 0 & $(1,1)$ & 0 & 0 \\
\hline$L_{2}$ & \multirow[t]{6}{*}{$b_{2}$} & $(1,2)$ & $-\frac{3}{2}$ & 0 & 0 & $\frac{1}{2}$ & 0 & 0 & $\frac{1}{2}$ & 0 & $(1,1)$ & 0 & 0 \\
\hline$Q_{2}$ & & $(3,2)$ & $\frac{1}{2}$ & 0 & 0 & $\frac{1}{2}$ & 0 & 0 & $-\frac{1}{2}$ & 0 & $(1,1)$ & 0 & 0 \\
\hline$d_{2}$ & & $(\overline{3}, 1)$ & $-\frac{1}{2}$ & -1 & 0 & $\frac{1}{2}$ & 0 & 0 & $-\frac{1}{2}$ & 0 & $(1,1)$ & 0 & 0 \\
\hline$N_{2}$ & & $(1,1)$ & $\frac{3}{2}$ & -1 & 0 & $\frac{1}{2}$ & 0 & 0 & $-\frac{1}{2}$ & 0 & $(1,1)$ & 0 & 0 \\
\hline$u_{2}$ & & $(\overline{3}, 1)$ & $-\frac{1}{2}$ & 1 & 0 & $\frac{1}{2}$ & 0 & 0 & $\frac{1}{2}$ & 0 & $(1,1)$ & 0 & 0 \\
\hline$e_{2}$ & & $(1,1)$ & $\frac{3}{2}$ & 1 & 0 & $\frac{1}{2}$ & 0 & 0 & $\frac{1}{2}$ & 0 & $(1,1)$ & 0 & 0 \\
\hline$L_{3}$ & \multirow[t]{6}{*}{$b_{3}$} & $(1,2)$ & $-\frac{3}{2}$ & 0 & 0 & 0 & $\frac{1}{2}$ & 0 & 0 & $\frac{1}{2}$ & $(1,1)$ & 0 & 0 \\
\hline$Q_{3}$ & & $(3,2)$ & $\frac{1}{2}$ & 0 & 0 & 0 & $\frac{1}{2}$ & 0 & 0 & $-\frac{1}{2}$ & $(1,1)$ & 0 & 0 \\
\hline$d_{3}$ & & $(\overline{3}, 1)$ & $-\frac{1}{2}$ & -1 & 0 & 0 & $\frac{1}{2}$ & 0 & 0 & $-\frac{1}{2}$ & $(1,1)$ & 0 & 0 \\
\hline$N_{3}$ & & $(1,1)$ & $\frac{3}{2}$ & -1 & 0 & 0 & $\frac{1}{2}$ & 0 & 0 & $-\frac{1}{2}$ & $(1,1)$ & 0 & 0 \\
\hline$u_{3}$ & & $(\overline{3}, 1)$ & $-\frac{1}{2}$ & 1 & 0 & 0 & $\frac{1}{2}$ & 0 & 0 & $\frac{1}{2}$ & $(1,1)$ & 0 & 0 \\
\hline$e_{3}$ & & $(1,1)$ & $\frac{3}{2}$ & 1 & 0 & 0 & $\frac{1}{2}$ & 0 & 0 & $\frac{1}{2}$ & $(1,1)$ & 0 & 0 \\
\hline$h_{1}$ & \multirow[t]{6}{*}{ NS } & $(1,2)$ & 0 & -1 & 1 & 0 & 0 & 0 & 0 & 0 & $(1,1)$ & 0 & 0 \\
\hline$h_{2}$ & & $(1,2)$ & 0 & -1 & 0 & 1 & 0 & 0 & 0 & 0 & $(1,1)$ & 0 & 0 \\
\hline$h_{3}$ & & $(1,2)$ & 0 & -1 & 0 & 0 & 1 & 0 & 0 & 0 & $(1,1)$ & 0 & 0 \\
\hline$\Phi_{12}$ & & $(1,1)$ & 0 & 0 & 1 & -1 & 0 & 0 & 0 & 0 & $(1,1)$ & 0 & 0 \\
\hline$\Phi_{13}$ & & $(1,1)$ & 0 & 0 & 1 & 0 & -1 & 0 & 0 & 0 & $(1,1)$ & 0 & 0 \\
\hline$\Phi_{23}$ & & $(1,1)$ & 0 & 0 & 0 & 1 & -1 & 0 & 0 & 0 & $(1,1)$ & 0 & 0 \\
\hline$h_{45}$ & \multirow{6}{*}{$\begin{array}{c}b_{1}+b_{2}+ \\
\alpha+\beta\end{array}$} & $(1,2)$ & 0 & -1 & $-\frac{1}{2}$ & $-\frac{1}{2}$ & 0 & 0 & 0 & 0 & $(1,1)$ & 0 & 0 \\
\hline$D_{45}$ & & $(3,1)$ & -1 & 0 & $-\frac{1}{2}$ & $-\frac{1}{2}$ & 0 & 0 & 0 & 0 & $(1,1)$ & 0 & 0 \\
\hline$\Phi_{45}$ & & $(1,1)$ & 0 & 0 & $-\frac{1}{2}$ & $-\frac{1}{2}$ & -1 & 0 & 0 & 0 & $(1,1)$ & 0 & 0 \\
\hline$\Phi_{1}^{ \pm}$ & & $(1,1)$ & 0 & 0 & $-\frac{1}{2}$ & $\frac{1}{2}$ & 0 & \pm 1 & 0 & 0 & $(1,1)$ & 0 & 0 \\
\hline$\Phi_{2}^{ \pm}$ & & $(1,1)$ & 0 & 0 & $-\frac{1}{2}$ & $\frac{1}{2}$ & 0 & 0 & \pm 1 & 0 & $(1,1)$ & 0 & 0 \\
\hline$\Phi_{3}^{ \pm}$ & & $(1,1)$ & 0 & 0 & $-\frac{1}{2}$ & $\frac{1}{2}$ & 0 & 0 & 0 & \pm 1 & $(1,1)$ & 0 & 0 \\
\hline
\end{tabular}

Table 1: Massless states for solution I (Ref. [13) which transform solely under the observable gauge group. $Q_{C}=3 / 2(B-L)$ and $Q_{L}=2 T_{3_{R}}$. In the NS and the $b_{1}+b_{2}+\alpha+\beta$ sectors contain also the conjugate states ( $\bar{h}_{1}$, etc.). The NS sector contains additional three singlet states, $\xi_{1,2,3}$, which are neutral under all the $U(1)$ symmetries. 


\begin{tabular}{|c|c|c|c|c|c|c|c|c|c|c|c|c|c|}
\hline$F$ & SEC & $S U(3) \times S U(2)$ & $Q_{C}$ & $Q_{L}$ & $Q_{1}$ & $Q_{2}$ & $Q_{3}$ & $Q_{4}$ & $Q_{5}$ & $Q_{6}$ & $S U(5) \times S U(3)$ & $Q_{7}$ & $Q_{8}$ \\
\hline$V_{1}$ & $b_{1}+2 \gamma+$ & $(1,1)$ & 0 & 0 & 0 & $\frac{1}{2}$ & $\frac{1}{2}$ & $\frac{1}{2}$ & 0 & 0 & $(1,3)$ & $-\frac{1}{2}$ & $\frac{5}{2}$ \\
\hline $\bar{V}_{1}$ & $(I)$ & $(1,1)$ & 0 & 0 & 0 & $\frac{1}{2}$ & $\frac{1}{2}$ & $\frac{1}{2}$ & 0 & 0 & $(1, \overline{3})$ & $\frac{1}{2}$ & $-\frac{5}{2}$ \\
\hline$T_{1}$ & & $(1,1)$ & 0 & 0 & 0 & $\frac{1}{2}$ & $\frac{1}{2}$ & $-\frac{1}{2}$ & 0 & 0 & $(5,1)$ & $-\frac{1}{2}$ & $-\frac{3}{2}$ \\
\hline $\bar{T}_{1}$ & & $(1,1)$ & 0 & 0 & 0 & $\frac{1}{2}$ & $\frac{1}{2}$ & $-\frac{1}{2}$ & 0 & 0 & $(\overline{5}, 1)$ & $\frac{1}{2}$ & $\frac{3}{2}$ \\
\hline$V_{2}$ & $b_{2}+2 \gamma+$ & $(1,1)$ & 0 & 0 & $\frac{1}{2}$ & 0 & $\frac{1}{2}$ & 0 & $\frac{1}{2}$ & 0 & $(1,3)$ & $-\frac{1}{2}$ & $\frac{5}{2}$ \\
\hline $\bar{V}_{2}$ & $(I)$ & $(1,1)$ & 0 & 0 & $\frac{1}{2}$ & 0 & $\frac{1}{2}$ & 0 & $\frac{1}{2}$ & 0 & $(1, \overline{3})$ & $\frac{1}{2}$ & $-\frac{5}{2}$ \\
\hline$T_{2}$ & & $(1,1)$ & 0 & 0 & $\frac{1}{2}$ & 0 & $\frac{1}{2}$ & 0 & $-\frac{1}{2}$ & 0 & $(5,1)$ & $-\frac{1}{2}$ & $-\frac{3}{2}$ \\
\hline $\bar{T}_{2}$ & & $(1,1)$ & 0 & 0 & $\frac{1}{2}$ & 0 & $\frac{1}{2}$ & 0 & $-\frac{1}{2}$ & 0 & $(\overline{5}, 1)$ & $\frac{1}{2}$ & $\frac{3}{2}$ \\
\hline$V_{3}$ & $b_{3}+\gamma+$ & $(1,1)$ & 0 & 0 & $\frac{1}{2}$ & $\frac{1}{2}$ & 0 & 0 & 0 & $\frac{1}{2}$ & $(1,3)$ & $-\frac{1}{2}$ & $\frac{5}{2}$ \\
\hline $\bar{V}_{3}$ & $(I)$ & $(1,1)$ & 0 & 0 & $\frac{1}{2}$ & $\frac{1}{2}$ & 0 & 0 & 0 & $\frac{1}{2}$ & $(1, \overline{3})$ & $\frac{1}{2}$ & $-\frac{5}{2}$ \\
\hline$T_{3}$ & & $(1,1)$ & 0 & 0 & $\frac{1}{2}$ & $\frac{1}{2}$ & 0 & 0 & 0 & $-\frac{1}{2}$ & $(5,1)$ & $-\frac{1}{2}$ & $-\frac{3}{2}$ \\
\hline $\bar{T}_{3}$ & & $(1,1)$ & 0 & 0 & $\frac{1}{2}$ & $\frac{1}{2}$ & 0 & 0 & 0 & $-\frac{1}{2}$ & $(\overline{5}, 1)$ & $\frac{1}{2}$ & $\frac{3}{2}$ \\
\hline$H_{1}$ & $b_{1}+b_{2}+$ & $(1,1)$ & $\frac{3}{4}$ & $\frac{1}{2}$ & $-\frac{1}{4}$ & $-\frac{1}{4}$ & $\frac{1}{4}$ & $-\frac{1}{2}$ & $-\frac{1}{2}$ & 0 & $(1,3)$ & $\frac{1}{4}$ & $-\frac{5}{4}$ \\
\hline $\mathrm{H}_{2}$ & $\alpha+\beta$ & $(1,1)$ & $-\frac{3}{4}$ & $-\frac{1}{2}$ & $\frac{1}{4}$ & $\frac{1}{4}$ & $-\frac{1}{4}$ & $-\frac{1}{2}$ & $-\frac{1}{2}$ & 0 & $(1, \overline{3})$ & $-\frac{1}{4}$ & $\frac{5}{4}$ \\
\hline$H_{3}$ & $\pm \gamma+(I)$ & $(1,1)$ & $\frac{3}{4}$ & $\frac{1}{2}$ & $-\frac{1}{4}$ & $-\frac{1}{4}$ & $\frac{1}{4}$ & $-\frac{1}{2}$ & $-\frac{1}{2}$ & 0 & $(1,1)$ & $-\frac{3}{4}$ & $\frac{15}{4}$ \\
\hline$H_{4}$ & & $(1,1)$ & $-\frac{3}{4}$ & $-\frac{1}{2}$ & $\frac{1}{4}$ & $\frac{1}{4}$ & $-\frac{1}{4}$ & $-\frac{1}{2}$ & $-\frac{1}{2}$ & 0 & $(1,1)$ & $\frac{3}{4}$ & $-\frac{15}{4}$ \\
\hline$H_{5}$ & $b_{1}+b_{3}+$ & $(1,1)$ & $\frac{3}{4}$ & $\frac{1}{2}$ & $-\frac{1}{4}$ & $\frac{1}{4}$ & $-\frac{1}{4}$ & $-\frac{1}{2}$ & 0 & $-\frac{1}{2}$ & $(1,3)$ & $\frac{1}{4}$ & $-\frac{5}{4}$ \\
\hline$H_{6}$ & $\alpha+\beta$ & $(1,1)$ & $-\frac{3}{4}$ & $-\frac{1}{2}$ & $\frac{1}{4}$ & $-\frac{1}{4}$ & $\frac{1}{4}$ & $-\frac{1}{2}$ & 0 & $-\frac{1}{2}$ & $(1, \overline{3})$ & $-\frac{1}{4}$ & $\frac{5}{4}$ \\
\hline$H_{7}$ & $\pm \gamma+(I)$ & $(1,1)$ & $\frac{3}{4}$ & $\frac{1}{2}$ & $-\frac{1}{4}$ & $\frac{1}{4}$ & $-\frac{1}{4}$ & $-\frac{1}{2}$ & 0 & $-\frac{1}{2}$ & $(1,1)$ & $-\frac{3}{4}$ & $\frac{15}{4}$ \\
\hline$H_{8}$ & & $(1,1)$ & $-\frac{3}{4}$ & $-\frac{1}{2}$ & $\frac{1}{4}$ & $-\frac{1}{4}$ & $\frac{1}{4}$ & $-\frac{1}{2}$ & 0 & $-\frac{1}{2}$ & $(1,1)$ & $\frac{3}{4}$ & $-\frac{15}{4}$ \\
\hline$H_{9}$ & $b_{2}+b_{3}+$ & $(1,1)$ & $\frac{3}{4}$ & $\frac{1}{2}$ & $\frac{1}{4}$ & $-\frac{1}{4}$ & $-\frac{1}{4}$ & 0 & $-\frac{1}{2}$ & $-\frac{1}{2}$ & $(1,3)$ & $\frac{1}{4}$ & $-\frac{5}{4}$ \\
\hline$H_{10}$ & $\alpha+\beta$ & $(1,1)$ & $-\frac{3}{4}$ & $-\frac{1}{2}$ & $-\frac{1}{4}$ & $-\frac{1}{4}$ & $\frac{1}{4}$ & 0 & $-\frac{1}{2}$ & $-\frac{1}{2}$ & $(1, \overline{3})$ & $-\frac{1}{4}$ & $\frac{5}{4}$ \\
\hline$H_{11}$ & $\pm \gamma+(I)$ & $(1,1)$ & $\frac{3}{4}$ & $\frac{1}{2}$ & $\frac{1}{4}$ & $-\frac{1}{4}$ & $-\frac{1}{4}$ & 0 & $-\frac{1}{2}$ & $-\frac{1}{2}$ & $(1,1)$ & $-\frac{3}{4}$ & $\frac{15}{4}$ \\
\hline$H_{12}$ & & $(1,1)$ & $-\frac{3}{4}$ & $-\frac{1}{2}$ & $-\frac{1}{4}$ & $-\frac{1}{4}$ & $\frac{1}{4}$ & 0 & $-\frac{1}{2}$ & $-\frac{1}{2}$ & $(1,1)$ & $\frac{3}{4}$ & $-\frac{15}{4}$ \\
\hline$H_{13}$ & $b_{1}+b_{3}+$ & $(1,1)$ & $-\frac{3}{4}$ & $\frac{1}{2}$ & $-\frac{1}{4}$ & $\frac{1}{4}$ & $-\frac{1}{4}$ & 0 & 0 & 0 & $(1,3)$ & $\frac{3}{4}$ & $\frac{5}{4}$ \\
\hline$H_{14}$ & $\alpha \pm \gamma+$ & $(1,1)$ & $\frac{3}{4}$ & $-\frac{1}{2}$ & $\frac{1}{4}$ & $-\frac{1}{4}$ & $\frac{1}{4}$ & 0 & 0 & 0 & $(1, \overline{3})$ & $-\frac{3}{4}$ & $-\frac{5}{4}$ \\
\hline$H_{15}$ & $(I)$ & $(1,2)$ & $-\frac{3}{4}$ & $-\frac{1}{2}$ & $-\frac{1}{4}$ & $\frac{1}{4}$ & $-\frac{1}{4}$ & 0 & 0 & 0 & $(1,1)$ & $-\frac{1}{4}$ & $-\frac{15}{4}$ \\
\hline$H_{16}$ & & $(1,2)$ & $\frac{3}{4}$ & $\frac{1}{2}$ & $\frac{1}{4}$ & $-\frac{1}{4}$ & $\frac{1}{4}$ & 0 & 0 & 0 & $(1,1)$ & $\frac{1}{4}$ & $\frac{15}{4}$ \\
\hline$H_{17}$ & & $(1,1)$ & $-\frac{3}{4}$ & $\frac{1}{2}$ & $-\frac{1}{4}$ & $-\frac{3}{4}$ & $-\frac{1}{4}$ & 0 & 0 & 0 & $(1,1)$ & $-\frac{1}{4}$ & $-\frac{15}{4}$ \\
\hline$H_{18}$ & & $(1,1)$ & $\frac{3}{4}$ & $-\frac{1}{2}$ & $\frac{1}{4}$ & $\frac{3}{4}$ & $\frac{1}{4}$ & 0 & 0 & 0 & $(1,1)$ & $\frac{1}{4}$ & $\frac{15}{4}$ \\
\hline
\end{tabular}

Table 2: Massless states for solution I (13]). 


\begin{tabular}{|c|c|c|rrrrrrrr|r|rr|}
\hline$F$ & SEC & $S U(3) \times S U(2)$ & $Q_{C}$ & $Q_{L}$ & $Q_{1}$ & $Q_{2}$ & $Q_{3}$ & $Q_{4}$ & $Q_{5}$ & $Q_{6}$ & $S U(5) \times S U(3)$ & $Q_{7}$ & $Q_{8}$ \\
\hline$H_{19}$ & $b_{2}+b_{3}+$ & $(1,1)$ & $-\frac{3}{4}$ & $\frac{1}{2}$ & $\frac{1}{4}$ & $-\frac{1}{4}$ & $-\frac{1}{4}$ & 0 & 0 & 0 & $(5,1)$ & $-\frac{1}{4}$ & $\frac{9}{4}$ \\
$H_{20}$ & $\alpha \pm \gamma+$ & $(1,1)$ & $\frac{3}{4}$ & $-\frac{1}{2}$ & $-\frac{1}{4}$ & $\frac{1}{4}$ & $\frac{1}{4}$ & 0 & 0 & 0 & $(\overline{5}, 1)$ & $\frac{1}{4}$ & $-\frac{9}{4}$ \\
$H_{21}$ & $(I)$ & $(3,1)$ & $\frac{1}{4}$ & $\frac{1}{2}$ & $\frac{1}{4}$ & $-\frac{1}{4}$ & $-\frac{1}{4}$ & 0 & 0 & 0 & $(1,1)$ & $-\frac{1}{4}$ & $-\frac{15}{4}$ \\
$H_{22}$ & & $(\overline{3}, 1)$ & $-\frac{1}{4}$ & $-\frac{1}{2}$ & $-\frac{1}{4}$ & $\frac{1}{4}$ & $\frac{1}{4}$ & 0 & 0 & 0 & $(1,1)$ & $\frac{1}{4}$ & $\frac{15}{4}$ \\
$H_{23}$ & & $(1,1)$ & $-\frac{3}{4}$ & $\frac{1}{2}$ & $\frac{1}{4}$ & $-\frac{1}{4}$ & $\frac{3}{4}$ & 0 & 0 & 0 & $(1,1)$ & $\frac{1}{4}$ & $\frac{15}{4}$ \\
$H_{24}$ & & $(1,1)$ & $\frac{3}{4}$ & $-\frac{1}{2}$ & $-\frac{1}{4}$ & $\frac{1}{4}$ & $-\frac{3}{4}$ & 0 & 0 & 0 & $(1,1)$ & $-\frac{1}{4}$ & $-\frac{15}{4}$ \\
$H_{25}$ & & $(1,1)$ & $-\frac{3}{4}$ & $\frac{1}{2}$ & $\frac{1}{4}$ & $\frac{3}{4}$ & $-\frac{1}{4}$ & 0 & 0 & 0 & $(1,1)$ & $-\frac{1}{4}$ & $-\frac{15}{4}$ \\
$H_{26}$ & & $(1,1)$ & $\frac{3}{4}$ & $-\frac{1}{2}$ & $-\frac{1}{4}$ & $-\frac{3}{4}$ & $\frac{1}{4}$ & 0 & 0 & 0 & $(1,1)$ & $\frac{1}{4}$ & $\frac{15}{4}$ \\
\hline$H_{27}$ & $b_{1}+b_{2}+$ & $(1,1)$ & $-\frac{3}{4}$ & $-\frac{1}{2}$ & $-\frac{1}{4}$ & $-\frac{1}{4}$ & $-\frac{1}{4}$ & $-\frac{1}{2}$ & $\frac{1}{2}$ & $\frac{1}{2}$ & $(1,3)$ & $\frac{1}{4}$ & $-\frac{5}{4}$ \\
$H_{28}$ & $b_{3}+\alpha+$ & $(1,1)$ & $\frac{3}{4}$ & $\frac{1}{2}$ & $\frac{1}{4}$ & $\frac{1}{4}$ & $\frac{1}{4}$ & $\frac{1}{2}$ & $-\frac{1}{2}$ & $-\frac{1}{2}$ & $(1, \overline{3})$ & $-\frac{1}{4}$ & $\frac{5}{4}$ \\
$H_{29}$ & $\beta \pm \gamma+$ & $(1,1)$ & $-\frac{3}{4}$ & $-\frac{1}{2}$ & $-\frac{1}{4}$ & $-\frac{1}{4}$ & $-\frac{1}{4}$ & $\frac{1}{2}$ & $-\frac{1}{2}$ & $\frac{1}{2}$ & $(1,1)$ & $-\frac{3}{4}$ & $\frac{15}{4}$ \\
$H_{30}$ & $(I)$ & $(1,1)$ & $\frac{3}{4}$ & $\frac{1}{2}$ & $\frac{1}{4}$ & $\frac{1}{4}$ & $\frac{1}{4}$ & $-\frac{1}{2}$ & $\frac{1}{2}$ & $-\frac{1}{2}$ & $(1,1)$ & $\frac{3}{4}$ & $-\frac{15}{4}$ \\
\hline
\end{tabular}

Table 3: Massless states for solution I ([13). 


\begin{tabular}{|c|c|c|c|c|c|c|c|c|c|c|c|c|}
\hline$F$ & SEC & $S U(4)_{C} \times S U(2)_{L}$ & $Q_{C^{\prime}}$ & $Q_{L}$ & $Q_{1}$ & $Q_{2}$ & $Q_{3}$ & $Q_{4^{\prime}}$ & $Q_{5^{\prime}}$ & $S U(5)_{H} \times S U(3)_{H}$ & $Q_{6}$ & $Q_{8^{\prime \prime}}$ \\
\hline$L_{1}$ & $b_{1} \oplus$ & $(1,2)$ & $-\frac{3}{2}$ & 0 & $\frac{1}{2}$ & 0 & 0 & $-\frac{1}{2}$ & $-\frac{1}{2}$ & $(1,1)$ & $-\frac{8}{3}$ & 0 \\
\hline$Q_{1}$ & $1+\alpha+2 \gamma$ & $(4,2)$ & $\frac{1}{2}$ & 0 & $\frac{1}{2}$ & 0 & 0 & $-\frac{1}{2}$ & $-\frac{1}{2}$ & $(1,1)$ & $-\frac{2}{3}$ & 0 \\
\hline$d_{1}$ & & $(\overline{4}, 1)$ & $-\frac{1}{2}$ & 1 & $\frac{1}{2}$ & 0 & 0 & $\frac{1}{2}$ & $\frac{1}{2}$ & $(1,1)$ & $\frac{2}{3}$ & 0 \\
\hline$N_{1}$ & & $(1,1)$ & $\frac{3}{2}$ & -1 & $\frac{1}{2}$ & 0 & 0 & $\frac{1}{2}$ & $\frac{1}{2}$ & $(1,1)$ & $\frac{8}{3}$ & 0 \\
\hline$e_{1}$ & & $(1,1)$ & $\frac{3}{2}$ & 1 & $\frac{1}{2}$ & 0 & 0 & $\frac{1}{2}$ & $\frac{1}{2}$ & $(1,1)$ & $\frac{8}{3}$ & 0 \\
\hline$u_{1}$ & & $(\overline{4}, 1)$ & $-\frac{1}{2}$ & -1 & $\frac{1}{2}$ & 0 & 0 & $\frac{1}{2}$ & $\frac{1}{2}$ & $(1,1)$ & $\frac{2}{3}$ & 0 \\
\hline$L_{2}$ & $b_{2} \oplus$ & $(1,2)$ & $-\frac{3}{2}$ & 0 & 0 & $\frac{1}{2}$ & 0 & $\frac{1}{2}$ & $-\frac{1}{2}$ & $(1,1)$ & $-\frac{8}{3}$ & 0 \\
\hline$Q_{2}$ & $1+\alpha+2 \gamma$ & $(4,2)$ & $\frac{1}{2}$ & 0 & 0 & $\frac{1}{2}$ & 0 & $\frac{1}{2}$ & $-\frac{1}{2}$ & $(1,1)$ & $-\frac{2}{3}$ & 0 \\
\hline$d_{2}$ & & $(\overline{4}, 1)$ & $-\frac{1}{2}$ & 1 & 0 & $\frac{1}{2}$ & 0 & $-\frac{1}{2}$ & $\frac{1}{2}$ & $(1,1)$ & $\frac{2}{3}$ & 0 \\
\hline$N_{2}$ & & $(1,1)$ & $\frac{3}{2}$ & -1 & 0 & $\frac{1}{2}$ & 0 & $-\frac{1}{2}$ & $\frac{1}{2}$ & $(1,1)$ & $\frac{8}{3}$ & 0 \\
\hline$e_{2}$ & & $(1,1)$ & $\frac{3}{2}$ & 1 & 0 & $\frac{1}{2}$ & 0 & $-\frac{1}{2}$ & $\frac{1}{2}$ & $(1,1)$ & $\frac{8}{3}$ & 0 \\
\hline$u_{2}$ & & $(\overline{4}, 1)$ & $-\frac{1}{2}$ & -1 & 0 & $\frac{1}{2}$ & 0 & $-\frac{1}{2}$ & $\frac{1}{2}$ & $(1,1)$ & $\frac{2}{3}$ & 0 \\
\hline$L_{3}$ & $b_{3} \oplus$ & $(1,2)$ & $-\frac{3}{2}$ & 0 & 0 & 0 & $\frac{1}{2}$ & 0 & 1 & $(1,1)$ & $-\frac{8}{3}$ & 0 \\
\hline$Q_{3}$ & $1+\alpha+2 \gamma$ & $(4,2)$ & $\frac{1}{2}$ & 0 & 0 & 0 & $\frac{1}{2}$ & 0 & 1 & $(1,1)$ & $-\frac{2}{3}$ & 0 \\
\hline$d_{3}$ & & $(\overline{4}, 1)$ & $-\frac{1}{2}$ & 1 & 0 & 0 & $\frac{1}{2}$ & 0 & -1 & $(1,1)$ & $\frac{2}{3}$ & 0 \\
\hline$N_{3}$ & & $(1,1)$ & $\frac{3}{2}$ & -1 & 0 & 0 & $\frac{1}{2}$ & 0 & -1 & $(1,1)$ & $\frac{8}{3}$ & 0 \\
\hline$e_{3}$ & & $(1,1)$ & $\frac{3}{2}$ & 1 & 0 & 0 & $\frac{1}{2}$ & 0 & -1 & $(1,1)$ & $\frac{8}{3}$ & 0 \\
\hline$u_{3}$ & & $(\overline{4}, 1)$ & $-\frac{1}{2}$ & -1 & 0 & 0 & $\frac{1}{2}$ & 0 & -1 & $(1,1)$ & $\frac{2}{3}$ & 0 \\
\hline$h_{1}$ & NS & $(1,2)$ & 0 & -1 & 1 & 0 & 0 & 0 & 0 & $(1,1)$ & c & 0 \\
\hline$h_{2}$ & & $(1,2)$ & 0 & -1 & 0 & 1 & 0 & 0 & 0 & $(1,1)$ & c & 0 \\
\hline$h_{3}$ & & $(1,2)$ & 0 & -1 & 0 & 0 & 1 & 0 & 0 & $(1,1)$ & c & 0 \\
\hline$\Phi_{12}$ & & $(1,1)$ & 0 & 0 & 1 & -1 & 0 & 0 & 0 & $(1,1)$ & c & 0 \\
\hline$\Phi_{13}$ & & $(1,1)$ & 0 & 0 & 1 & 0 & -1 & 0 & 0 & $(1,1)$ & c & 0 \\
\hline$\Phi_{23}$ & & $(1,1)$ & 0 & 0 & 0 & 1 & -1 & 0 & 0 & $(1,1)$ & c & 0 \\
\hline$h_{45}$ & $b_{1}+b_{2}+$ & $(1,2)$ & 0 & -1 & $\frac{1}{2}$ & $\frac{1}{2}$ & 0 & 0 & 0 & $(1,1)$ & c & 0 \\
\hline$h_{45}^{\prime}$ & $\alpha+\beta$ & $(1,2)$ & 0 & -1 & $-\frac{1}{2}$ & $-\frac{1}{2}$ & 0 & 0 & 0 & $(1,1)$ & c & 0 \\
\hline$\Phi_{45}$ & & $(1,1)$ & 0 & 0 & $-\frac{1}{2}$ & $-\frac{1}{2}$ & -1 & 0 & 0 & $(1,1)$ & $\frac{2}{3}$ & 0 \\
\hline$\Phi_{45}^{\prime}$ & & $(1,1)$ & 0 & 0 & $-\frac{1}{2}$ & $-\frac{1}{2}$ & 1 & 0 & 0 & $(1,1)$ & c & 0 \\
\hline$\Phi 1,2$ & & $(1,1)$ & 0 & 0 & $-\frac{1}{2}$ & $\frac{1}{2}$ & 0 & 0 & 0 & $(1,1)$ & c & 0 \\
\hline
\end{tabular}

Table 4: Massless states for solution II (ref. [14]). 


\begin{tabular}{|c|c|c|c|c|c|c|c|c|c|c|c|c|}
\hline$F$ & SEC & $S U(4)_{C} \times S U(2)_{L}$ & $Q_{C^{\prime}}$ & $Q_{L}$ & $Q_{1}$ & $Q_{2}$ & $Q_{3}$ & $Q_{4^{\prime}}$ & $Q_{5^{\prime}}$ & $S U(5)_{H} \times S U(3)_{H}$ & $Q_{6^{\prime}}$ & $Q_{8^{\prime \prime}}$ \\
\hline$V_{1}$ & $b_{1}+2 \gamma$ & $(1,1)$ & $-\frac{1}{2}$ & 0 & 0 & $\frac{1}{2}$ & $\frac{1}{2}$ & $\frac{1}{2}$ & $\frac{1}{2}$ & $(1,3)$ & $\frac{8}{3}$ & $\frac{5}{2}$ \\
\hline $\bar{V}_{1}$ & & $(1,1)$ & $\frac{1}{2}$ & 0 & 0 & $\frac{1}{2}$ & $\frac{1}{2}$ & $-\frac{1}{2}$ & $-\frac{1}{2}$ & $(1, \overline{3})$ & $-\frac{8}{3}$ & $-\frac{5}{2}$ \\
\hline$T_{1}$ & & $(1,1)$ & $\frac{1}{2}$ & 0 & 0 & $\frac{1}{2}$ & $\frac{1}{2}$ & $-\frac{1}{2}$ & $-\frac{1}{2}$ & $(5,1)$ & $-\frac{8}{3}$ & $\frac{3}{2}$ \\
\hline $\bar{T}_{1}$ & & $(1,1)$ & $-\frac{1}{2}$ & 0 & 0 & $\frac{1}{2}$ & $\frac{1}{2}$ & $\frac{1}{2}$ & $\frac{1}{2}$ & $(\overline{5}, 1)$ & $\frac{8}{3}$ & $-\frac{3}{2}$ \\
\hline$V_{2}$ & $b_{2}+2 \gamma$ & $(1,1)$ & $-\frac{1}{2}$ & 0 & $\frac{1}{2}$ & 0 & $\frac{1}{2}$ & $-\frac{1}{2}$ & $\frac{1}{2}$ & $(1,3)$ & $\frac{8}{3}$ & $\frac{5}{2}$ \\
\hline $\bar{V}_{2}$ & & $(1,1)$ & $\frac{1}{2}$ & 0 & $\frac{1}{2}$ & 0 & $\frac{1}{2}$ & $\frac{1}{2}$ & $-\frac{1}{2}$ & $(1, \overline{3})$ & $-\frac{8}{3}$ & $-\frac{5}{2}$ \\
\hline$T_{2}$ & & $(1,1)$ & $\frac{1}{2}$ & 0 & $\frac{1}{2}$ & 0 & $\frac{1}{2}$ & $\frac{1}{2}$ & $-\frac{1}{2}$ & $(5,1)$ & $-\frac{8}{3}$ & $\frac{3}{2}$ \\
\hline $\bar{T}_{2}$ & & $(1,1)$ & $-\frac{1}{2}$ & 0 & $\frac{1}{2}$ & 0 & $\frac{1}{2}$ & $-\frac{1}{2}$ & $\frac{1}{2}$ & $(\overline{5}, 1)$ & $\frac{8}{3}$ & $-\frac{3}{2}$ \\
\hline$V_{3}$ & $b_{3}+2 \gamma$ & $(1,1)$ & $-\frac{1}{2}$ & 0 & $\frac{1}{2}$ & $\frac{1}{2}$ & 0 & 0 & -1 & $(1,3)$ & $\frac{8}{3}$ & $\frac{5}{2}$ \\
\hline $\bar{V}_{3}$ & & $(1,1)$ & $\frac{1}{2}$ & 0 & $\frac{1}{2}$ & $\frac{1}{2}$ & 0 & 0 & 1 & $(1, \overline{3})$ & $-\frac{8}{3}$ & $-\frac{5}{2}$ \\
\hline$T_{3}$ & & $(1,1)$ & $\frac{1}{2}$ & 0 & $\frac{1}{2}$ & $\frac{1}{2}$ & 0 & 0 & 1 & $(5,1)$ & $-\frac{8}{3}$ & $\frac{3}{2}$ \\
\hline $\bar{T}_{3}$ & & $(1,1)$ & $-\frac{1}{2}$ & 0 & $\frac{1}{2}$ & $\frac{1}{2}$ & 0 & 0 & -1 & $(\overline{5}, 1)$ & $\frac{8}{3}$ & $-\frac{3}{2}$ \\
\hline$l_{1}$ & $b_{2}+b_{3}+$ & $(1,2)$ & $-\frac{3}{4}$ & $-\frac{1}{2}$ & $\frac{1}{4}$ & $-\frac{1}{4}$ & $-\frac{1}{4}$ & 0 & 0 & $(1,1)$ & 0 & $-\frac{15}{4}$ \\
\hline $\bar{l}_{1}$ & $\beta+\gamma+\xi$ & $(1, \overline{2})$ & $\frac{3}{4}$ & $\frac{1}{2}$ & $-\frac{1}{4}$ & $\frac{1}{4}$ & $\frac{1}{4}$ & 0 & 0 & $(1,1)$ & 0 & $\frac{15}{4}$ \\
\hline$S_{1}$ & & $(1,1)$ & $\frac{3}{4}$ & $-\frac{1}{2}$ & $-\frac{3}{4}$ & $-\frac{1}{4}$ & $-\frac{1}{4}$ & 0 & 0 & $(1,1)$ & 0 & $-\frac{15}{4}$ \\
\hline $\bar{S}_{1}$ & & $(1,1)$ & $-\frac{3}{4}$ & $\frac{1}{2}$ & $\frac{3}{4}$ & $\frac{1}{4}$ & $\frac{1}{4}$ & 0 & 0 & $(1,1)$ & 0 & $\frac{15}{4}$ \\
\hline$S_{2}$ & & $(1,1)$ & $\frac{3}{4}$ & $-\frac{1}{2}$ & $-\frac{1}{4}$ & $-\frac{3}{4}$ & $\frac{1}{4}$ & 0 & 0 & $(1,1)$ & 0 & $-\frac{15}{4}$ \\
\hline $\bar{S}_{2}$ & & $(1,1)$ & $-\frac{3}{4}$ & $\frac{1}{2}$ & $\frac{1}{4}$ & $\frac{3}{4}$ & $-\frac{1}{4}$ & 0 & 0 & $(1,1)$ & 0 & $\frac{15}{4}$ \\
\hline$S_{3}$ & & $(1,1)$ & $\frac{3}{4}$ & $-\frac{1}{2}$ & $-\frac{1}{4}$ & $\frac{1}{4}$ & $-\frac{3}{4}$ & 0 & 0 & $(1,1)$ & 0 & $-\frac{15}{4}$ \\
\hline $\bar{S}_{3}$ & & $(1,1)$ & $-\frac{3}{4}$ & $\frac{1}{2}$ & $\frac{1}{4}$ & $-\frac{1}{4}$ & $\frac{3}{4}$ & 0 & 0 & $(1,1)$ & 0 & $\frac{15}{4}$ \\
\hline$H_{1}$ & & $(1,1)$ & $-\frac{3}{4}$ & $\frac{1}{2}$ & $\frac{1}{4}$ & $-\frac{1}{4}$ & $-\frac{1}{4}$ & 0 & 0 & $(5,1)$ & 0 & $\frac{9}{4}$ \\
\hline $\bar{H}_{1}$ & & $(1,1)$ & $\frac{3}{4}$ & $-\frac{1}{2}$ & $-\frac{1}{4}$ & $\frac{1}{4}$ & $\frac{1}{4}$ & 0 & 0 & $(\overline{5}, 1)$ & 0 & $-\frac{9}{4}$ \\
\hline$l_{2}$ & $b_{1}+b_{3}+$ & $(1,2)$ & $-\frac{3}{4}$ & $-\frac{1}{2}$ & $\frac{1}{4}$ & $-\frac{1}{4}$ & $-\frac{1}{4}$ & 0 & 0 & $(1,1)$ & 0 & $-\frac{15}{4}$ \\
\hline $\bar{l}_{2}$ & $\alpha+\gamma+\xi$ & $(1, \overline{2})$ & $\frac{3}{4}$ & $\frac{1}{2}$ & $-\frac{1}{4}$ & $\frac{1}{4}$ & $\frac{1}{4}$ & 0 & 0 & $(1,1)$ & 0 & $\frac{15}{4}$ \\
\hline$S_{4}$ & & $(1,1)$ & $\frac{3}{4}$ & $-\frac{1}{2}$ & $-\frac{3}{4}$ & $-\frac{1}{4}$ & $\frac{1}{4}$ & 0 & 0 & $(1,1)$ & 0 & $-\frac{15}{4}$ \\
\hline $\bar{S}_{4}$ & & $(1,1)$ & $-\frac{3}{4}$ & $\frac{1}{2}$ & $\frac{3}{4}$ & $\frac{1}{4}$ & $-\frac{1}{4}$ & 0 & 0 & $(1,1)$ & 0 & $\frac{15}{4}$ \\
\hline$S_{5}$ & & $(1,1)$ & $\frac{3}{4}$ & $-\frac{1}{2}$ & $-\frac{1}{4}$ & $-\frac{3}{4}$ & $-\frac{1}{4}$ & 0 & 0 & $(1,1)$ & 0 & $-\frac{15}{4}$ \\
\hline $\bar{S}_{5}$ & & $(1,1)$ & $-\frac{3}{4}$ & $\frac{1}{2}$ & $\frac{1}{4}$ & $\frac{3}{4}$ & $\frac{1}{4}$ & 0 & 0 & $(1,1)$ & 0 & $\frac{15}{4}$ \\
\hline$S_{6}$ & & $(1,1)$ & $\frac{3}{4}$ & $-\frac{1}{2}$ & $\frac{1}{4}$ & $-\frac{1}{4}$ & $-\frac{3}{4}$ & 0 & 0 & $(1,1)$ & 0 & $-\frac{15}{4}$ \\
\hline $\bar{S}_{6}$ & & $(1,1)$ & $-\frac{3}{4}$ & $\frac{1}{2}$ & $-\frac{1}{4}$ & $\frac{1}{4}$ & $\frac{3}{4}$ & 0 & 0 & $(1,1)$ & 0 & $\frac{15}{4}$ \\
\hline$H_{2}$ & & $(1,1)$ & $-\frac{3}{4}$ & $\frac{1}{2}$ & $-\frac{1}{4}$ & $\frac{1}{4}$ & $-\frac{1}{4}$ & 0 & 0 & $(5,1)$ & 0 & $\frac{9}{4}$ \\
\hline $\bar{H}_{2}$ & & $(1,1)$ & $\frac{3}{4}$ & $-\frac{1}{2}$ & $\frac{1}{4}$ & $-\frac{1}{4}$ & $\frac{1}{4}$ & 0 & 0 & $(\overline{5}, 1)$ & 0 & $-\frac{9}{4}$ \\
\hline
\end{tabular}

Table 5: Massless states for solution II (ref. [14]). 


\begin{tabular}{|c|c|c|rrrrrrr|c|rr|}
\hline$F$ & SEC & $S U(4)_{C} \times S U(2)_{L}$ & $Q_{C^{\prime}}$ & $Q_{L}$ & $Q_{1}$ & $Q_{2}$ & $Q_{3}$ & $Q_{4^{\prime}}$ & $Q_{5^{\prime}}$ & $S U(5)_{H} \times S U(3)_{H}$ & $Q_{6^{\prime}}$ & $Q_{8^{\prime \prime}}$ \\
\hline$l_{4}$ & $1+b_{1}+$ & $(1,2)$ & -1 & 0 & $-\frac{1}{2}$ & 0 & 0 & $-\frac{1}{2}$ & $-\frac{1}{2}$ & $(1,1)$ & $\frac{16}{3}$ & 0 \\
$S_{7}$ & $\alpha+2 \gamma$ & $(1,1)$ & 1 & 1 & $-\frac{1}{2}$ & 0 & 0 & $\frac{1}{2}$ & $\frac{1}{2}$ & $(1,1)$ & $-\frac{16}{3}$ & 0 \\
$\bar{S}_{7}$ & & $(1,1)$ & 1 & -1 & $-\frac{1}{2}$ & 0 & 0 & $\frac{1}{2}$ & $\frac{1}{2}$ & $(1,1)$ & $-\frac{16}{3}$ & 0 \\
\hline$l_{5}$ & $1+b_{2}+$ & $(1,2)$ & -1 & 0 & 0 & $-\frac{1}{2}$ & 0 & $\frac{1}{2}$ & $-\frac{1}{2}$ & $(1,1)$ & $\frac{16}{3}$ & 0 \\
$S_{8}$ & $\alpha+2 \gamma$ & $(1,1)$ & 1 & 1 & 0 & $-\frac{1}{2}$ & 0 & $-\frac{1}{2}$ & $\frac{1}{2}$ & $(1,1)$ & $-\frac{16}{3}$ & 0 \\
$\bar{S}_{8}$ & & $(1,1)$ & 1 & -1 & 0 & $-\frac{1}{2}$ & 0 & $-\frac{1}{2}$ & $\frac{1}{2}$ & $(1,1)$ & $-\frac{16}{3}$ & 0 \\
\hline$l_{6}$ & $1+b_{3}+$ & $(1,2)$ & -1 & 0 & 0 & 0 & $-\frac{1}{2}$ & 0 & 1 & $(1,1)$ & $\frac{16}{3}$ & 0 \\
$S_{9}$ & $\alpha+2 \gamma$ & $(1,1)$ & 1 & 1 & 0 & 0 & $-\frac{1}{2}$ & 0 & -1 & $(1,1)$ & $-\frac{16}{3}$ & 0 \\
$\bar{S}_{9}$ & & $(1,1)$ & 1 & -1 & 0 & 0 & $-\frac{1}{2}$ & 0 & -1 & $(1,1)$ & $-\frac{16}{3}$ & 0 \\
\hline$S_{10}$ & $1+s+$ & $(1,1)$ & -2 & 0 & 0 & 0 & 0 & -1 & -1 & $(1,1)$ & $-\frac{4}{3}$ & 0 \\
$\bar{S}_{10}$ & $\alpha+2 \gamma$ & $(1,1)$ & -2 & 0 & 0 & 0 & 0 & 1 & 1 & $(1,1)$ & $\frac{4}{3}$ & 0 \\
\hline
\end{tabular}

Table 6: Massless states for solution II ([14). 